\title{
A coding of separable Banach spaces. Analytic and coanalytic families of Banach spaces
}

\author{
by
}

\author{
Benoît Bossard (Paris)
}

\begin{abstract}
When the set of closed subspaces of $C(\Delta)$, where $\Delta$ is the Cantor set, is equipped with the standard Effros-Borel structure, the graph of the basic relations between Banach spaces (isomorphism, being isomorphic to a subspace, quotient, direct sum...) is analytic non-Borel. Many natural families of Banach spaces (such as reflexive spaces, spaces not containing $\ell_{1}(\omega), \ldots$ ) are coanalytic non-Borel. Some natural ranks (rank of embedding, Szlenk indices) are shown to be coanalytic ranks. Applications are given to universality questions. Analogous results are shown for basic sequences modulo equivalence.
\end{abstract}

0. Introduction. Classifying Banach spaces is notoriously difficult, and it is natural to conjecture that the obstruction to such a classification lies in the topological complexity of the relevant relations, and in particular of the isomorphism equivalence relation. To support this conjecture, one needs of course a natural and usable frame in which such topological notions can be handled. The purpose of the present work is to provide such a frame.

The collection of separable Banach spaces is not a set, and we first need a proper parametrization of this collection. We choose to consider it as the set of all closed subspaces of the space $C(\Delta)$ of continuous functions on the Cantor set. It is indeed well known that every separable Banach space is isometric to a subspace of $C(\Delta)$. This choice could be considered as arbitrary; however we show that natural but different choices lead to the same levels of complexity.

To investigate the topological complexity of natural families of Banach spaces, we use the theory of analytic sets, introduced by Suslin and Lusin. The basic results of this theory are presented e.g. in [K-L1], [K] or [Z]. There is a strong interplay between analytic sets and classical analysis, for which

2000 Mathematics Subject Classification: Primary 46B20.

Key words and phrases: codings, analytic relations, coanalytic sets, coanalytic ranks. 
we refer for instance to the classical results of S. Mazurkiewicz $[\mathrm{M}]$ and W. Hurewicz $[\mathrm{H}]$, and for more recent results to [Mau], [Bou1], [Ka1], [Ka2], [G1], [D-G-S], [B-G-K], [Ko] and finally to [K-L1] and $[\mathrm{K}]$ and references therein.

The gist of our results is that the natural relations, and natural classes of Banach spaces, are as complicated as they look at first sight: for instance, the isomorphism relation is analytic non-Borel, the class of spaces which do not contain an isomorphic copy of a given space is coanalytic non-Borel, the isomorphism class of a given space is in general non-Borel. Several consequences are spelled out: for instance, there is no constructive way to pick a representative in each isomorphism class. Applications are given to universality questions: indeed when a property defines a coanalytic non-Borel class (it is so e.g. for reflexivity, separability of the dual, and many others), it cannot reduce to being isomorphic to a subspace of a given space since the latter is an analytic class. We show moreover that classical tools of descriptive set theory are well adjusted to Banach space theory: for instance, classical indices such as the Szlenk index turn out to be coanalytic ranks. This leads to applications of Kunen-Martin's transfinite "uniform boundedness" principle.

Let us summarize the content of this paper. Notation and preliminaries are given below in Section 0. In Section 1, we use techniques from [J1], [L-S] and $[\mathrm{P}]$ to construct two families of Banach spaces $U_{1}(\theta)$ and $U_{2}(\theta)$, indexed by the trees $\theta$ on $\omega$, such that if $\theta$ is well founded then $U_{1}(\theta)$ does not contain any reflexive infinite-dimensional subspace and $U_{2}(\theta)$ is reflexive, and if not $U_{1}(\theta)$ and $U_{2}(\theta)$ are universal for the separable Banach spaces.

Section 2 is devoted to codings of separable Banach spaces up to isomorphisms by standard Borel spaces. This can be done by a proper use of the Effros-Borel structure. We show that the classical Banach space notions such as isomorphism, being isomorphic to a subspace, quotient, direct sum lead to analytic non-Borel relations, and that the isomorphism relation has no analytic section. Therefore, the isomorphism relation is not smooth (in the sense of $[\mathrm{H}-\mathrm{K}-\mathrm{L}])$ and there are no Borel calculable invariants which classify separable Banach spaces up to isomorphism. We also observe that natural but different ways to code the separable Banach spaces yield the same complexity results.

In Section 3, many natural families of separable Banach spaces are shown to be coanalytic non-Borel in the sense of Section 2: reflexive spaces, spaces with separable dual, spaces which do not contain $\ell_{1}(\omega)$, spaces which are not universal, spaces with RNP. The family of separable Banach spaces with non-separable dual and which do not contain $\ell_{1}(\omega)$ is a difference of two coanalytic families but is neither coanalytic nor analytic. Some new results on universal spaces are obtained as applications. 
In Section 4, natural ranks are shown to be coanalytic ranks on some of the families studied in Section 3: ranks of embedding, Szlenk indices. This allows us to show that the Szlenk index and the dentability index are equivalent for spaces with separable duals, and that the Szlenk index and the rank of embedding of $\ell_{1}$ are equivalent for subspaces of spaces with an unconditional basis.

Section 5 undertakes a similar study of coding basic sequences up to equivalence: the relation of equivalence between bases is a Borel relation. Using Bellenot's space [Be], we show that this relation has no analytic sections. The family of shrinking basic sequences and the family of boundedly complete basic sequences are coanalytic non-Borel, with natural coanalytic ranks.

Finally, Section 6 uses Gowers' solution [Gow] to the hyperplane problem to provide an embedding of the equivalence relation $E_{0}$ into the isomorphism relation between separable Banach spaces. This gives an improvement of the result of Section 5 obtained through Bellenot's space. The existence of such an embedding is natural in view of $[\mathrm{H}-\mathrm{K}-\mathrm{L}]$. However, some extra work is needed since the isomorphism relation is analytic non-Borel.

Acknowledgements. Most of the results of this paper are part of a thesis [Bos] prepared under the supervision of G. Godefroy at the University of Paris 6 . The author would like to thank G. Godefroy for his suggestions and encouragement.

Notations and preliminaries. We denote by $\omega=\{0,1,2, \ldots\}$ the first infinite ordinal, by $\omega^{\star}$ the set $\omega \backslash\{0\}$, by $\omega_{1}$ the first uncountable ordinal. Let $A$ be a set. We denote by $\mathcal{P}(A)$ the set of subsets of $A$, and by $A^{\omega}$ (resp. $A^{<\omega}$ ) the set of all infinite (resp. finite) sequences in $A$. If $\underline{x} \in A^{\omega}$, we write $\underline{x}=\left(x_{i}\right)_{i}$. Concatenation is denoted by $\frown$, and if $B \subseteq A^{<\omega}$ or $B \subseteq A^{\omega}$, and $s \in A^{<\omega}$, we denote by $s \frown B$ the set $\{s \frown t ; t \in B\}$.

Let $X$ be a Banach space. Then $B_{X}$ is its closed unit ball, and for $x \in X$ and $\varepsilon>0, B(x, \varepsilon)=\{y \in X ;\|y-x\|<\varepsilon\}$. For $A \subseteq X, \operatorname{conv}(A)$ denotes its convex hull, $\operatorname{sp}(A)\left(\right.$ resp. $\left.\operatorname{sp}_{\mathbb{Q}}(A)\right)$ the real vector (resp. $\mathbb{Q}$-vector) space spanned by $A, \overline{\operatorname{conv}}(A)$ and $\overline{\operatorname{sp}}(A)$ their closures; $A^{\perp}$ is the orthogonal complement of $A$ and $\operatorname{diam}(A)=\sup \{\|x-y\| ; x, y \in A\}$. If $A \subseteq X^{\star}$, then $\bar{A}^{\star}$ denotes its $w^{\star}$-closure. If $\underline{\lambda}$ and $\underline{x}$ are finite or infinite sequences respectively in $\mathbb{R}$ and $X$, we will write $\underline{\lambda} \cdot \underline{x}=\sum_{i} \lambda_{i} x_{i}$. If $\underline{x} \in X^{\omega}, \underline{y} \in Y^{\omega}$ where $Y$ is a Banach space, and $k \in[1,+\infty)$, then $\underline{x} \sim_{k} \underline{y}$ means: for all $\underline{\lambda} \in \mathbb{R}^{<\omega}$, $k^{-1}\|\underline{\lambda} \cdot \underline{x}\| \leq\|\underline{\lambda} \cdot \underline{y}\| \leq k\|\underline{\lambda} \cdot \underline{x}\|$, and we will write $\underline{x} \sim \underline{y}$ if there exists some $k \in[1,+\infty)$ such that $\underline{x} \sim_{k} \underline{y}$. The notations $X \simeq Y, X \subset Y, Y \Rightarrow X$ will mean respectively: $X$ and $Y$ are isomorphic, $X$ is isomorphic to a subspace of $Y, X$ is isomorphic to a quotient space of $Y$. The inclusion of sets is denoted by $\subseteq$. 
Let $P$ be a Polish space, and $\mathcal{O}$ a basis of open subsets of $P$. We denote by $\mathcal{F}(P)$ the set of all closed subsets of $P$ equipped with the Effros-Borel structure (i.e. the canonical Borel structure generated by the family $\{\{F \in$ $\mathcal{F}(P) ; F \cap O \neq \emptyset\} ; O \in \mathcal{O}\}$ \} (see $[\mathrm{C}]$ ). If in addition $P$ is compact, the Effros-Borel structure is generated by the Hausdorff topology, thus by the family $\{\{F \in \mathcal{F}(P) ; F \subseteq O\} ; O \in \mathcal{O}\}$.

Let $P$ be a standard Borel space (i.e. the Borel structure is generated by a Polish topology). We refer to [K-L1] and [C] for the following notions and properties. A subset $C$ of $P$ is analytic if it is the Borel image of a Borel subset of a Polish space, coanalytic if $P \backslash C$ is analytic, and Borel if it is both analytic and coanalytic (this is Suslin's separation theorem). When $C$ is coanalytic, there exists a coanalytic rank on $C$, that is to say, a function $\sigma: P \rightarrow\left[0, \omega_{1}\right]$ such that $C=\left\{x ; \sigma(x)<\omega_{1}\right\}$ and the relations " $x \in C$ and $\sigma(x) \leq \sigma(y)$ " and " $x \in C$ and $\sigma(x)<\sigma(y)$ " are both coanalytic in $P^{2}$. Some properties of coanalytic ranks are summarized in the following proposition.

Proposition 0.1. Let $\sigma$ be a coanalytic rank on a coanalytic subset $C$ of a standard Borel space $P$.

(i) For every $\alpha<\omega_{1}, B_{\alpha}=\{x \in C ; \sigma(x) \leq \alpha\}$ is Borel.

(ii) If $A \subseteq C$ is analytic, then $A \subseteq B_{\alpha}$ for some $\alpha<\omega_{1}$.

(iii) If $\sigma^{\prime}$ is another coanalytic rank on $C$, then there exists $\psi: \omega_{1} \rightarrow \omega_{1}$ such that if $\alpha<\omega_{1}$ and if $x \in C$ is such that $\sigma(x) \leq \alpha$, then $\sigma^{\prime}(x) \leq \psi(\alpha)$.

(iv) Let $A$ be a coanalytic subset of a standard Borel space $P^{\prime}$. Assume there is a Borel map $\varphi: P^{\prime} \rightarrow P$ such that $\varphi^{-1}(C)=A$. Then $\sigma \circ \varphi$ is a coanalytic rank on the coanalytic subset $A$.

We refer to [K-L1] for (i), (ii) and (iv), which are classical properties of coanalytic ranks; (iii) follows from (i) and (ii).

We refer again to [K-L1] for the definition of tree, height, branch. The height of a tree $\theta$ is denoted by $\operatorname{ht}(\theta)$. The tree $\omega^{<\omega}$ of finite sequences in $\omega$ will be denoted by $T$, and the set of trees on $\omega$, i.e. the set of subtrees of $T$, is denoted by $\mathcal{T}$. Let $s \in T$; its length is denoted by $|s|$. If $t \in T$ and $|s| \leq|t|$ (or if $t$ is a branch of $T$ ) and if " $s$ begins $t$ ", we will write $s \preceq t$. If $s \preceq t$ and $s \neq t$, we will write $s \prec t$. When $t \in T$ and $s \preceq t$, the interval $[s, t]$ is the set $\{w \in T ; s \preceq w \preceq t\}$. We fix an enumeration $\mathcal{K}: \omega \rightarrow \omega^{<\omega}$ of $\omega^{<\omega}$ such that if $s \prec s^{\prime}$, then $\bar{s}<\bar{s}^{\prime}$, where $\bar{s}=\mathcal{K}^{-1}(s)$. We set $s_{n}=\mathcal{K}(n)$.

We denote by WF the subset of $\mathcal{T}$ consisting of well founded trees, i.e. of trees which have no (infinite) branch, and $\mathrm{MF}=\mathcal{T} \backslash \mathrm{WF}$. It is classical that $\mathrm{WF}$ is a complete coanalytic subset, that is to say, WF is coanalytic and for any Polish space and any coanalytic subset $\mathcal{Q}$ of $P$, there is a Borel function $f: P \rightarrow \mathcal{T}$ such that $\mathcal{Q}=f^{-1}(\mathrm{WF})$. It is classical that a complete coanalytic subset is not analytic, thus not Borel. A coanalytic rank on WF is given by the map $\theta \mapsto h t(\theta)$. 
A Banach space is universal for separable Banach spaces (for short, universal) if it contains an isomorphic copy of every separable Banach space. It is well known that the space $C(\Delta)$ of all continuous functions on the Cantor set $\Delta=2^{\omega}$ is universal.

Let $E$ be an equivalence relation on a set $A$. A section $S$ of $E$ is a subset of $A$ such that for every $x \in A$, there is one and only one $y \in S$ such that $x E y$.

1. Construction of the families $\left\{U_{1}(\theta) ; \theta \in \mathcal{T}\right\}$ and $\left\{U_{2}(\theta) ; \theta \in \mathcal{T}\right\}$. In this section, we associate to any tree $\theta \in \mathcal{T}$ two separable Banach spaces $U_{1}(\theta)$ and $U_{2}(\theta)$ which are universal if $\theta$ is not well founded, and such that $U_{1}(\theta)$ has no infinite-dimensional reflexive subspaces and $U_{2}(\theta)$ is reflexive if $\theta$ is well founded.

We use the universal Banach space $U$ built by Pełczyński ([P] or [L-T1], pp. 92-93). Let us recall some of its properties.

THEOREM 1.1. There exists a universal separable Banach space $U$ with a basis $\underline{u}=\left(u_{i}\right)_{i \in \omega}$ such that for any basic sequence $\left(x_{k}\right)_{k \in \omega}$, there is a subsequence $\left(u_{n_{k}}\right)_{k \in \omega}$ of $\underline{u}$ which is equivalent to $\left(x_{k}\right)_{k \in \omega}$ and complemented; that is to say, the natural projection $\Pi$, defined by $\Pi\left(u_{n_{k}}\right)=u_{n_{k}}$ for any $k \in \omega$ and $\Pi\left(u_{n}\right)=0$ if $n \neq\left\{n_{k} ; k \in \omega\right\}$, is bounded.

Moreover every separable Banach space with a basis which contains isomorphic copies of all separable Banach spaces with a basis as complemented subspaces must be isomorphic to $U$.

We now follow the lines of the construction of the James tree spaces ([J1]; see [L-S]).

We denote by $c_{00}(T)$ the space of finitely supported functions from $T=$ $\omega^{<\omega}$ to $\mathbb{R}$, and by $\chi_{s}: T \rightarrow\{0,1\}$ the characteristic function of $\{s\}$ for every $s \in T$. Thus $c_{00}(T)=\operatorname{sp}\left(\left\{\chi_{s} ; s \in T\right\}\right)$.

An admissible choice of intervals is a finite set $\left\{I_{j} ; 0 \leq j \leq k\right\}$ of intervals of $T$ such that every branch of $T$ meets at most one of these intervals. We define the following norms on $c_{00}(T)$ :

$$
\begin{aligned}
& \|y\|_{1}=\sup \left(\sum_{j=0}^{k}\left\|\sum_{s \in I_{j}} y(s) u_{|s|}\right\|\right), \\
& \|y\|_{2}=\sup \left(\left(\sum_{j=0}^{k}\left\|\sum_{s \in I_{j}} y(s) u_{|s|}\right\|^{2}\right)^{1 / 2}\right),
\end{aligned}
$$

where $|s|$ is the length of $s \in T$ and where the supremum is taken over $k \in \omega$ and over all admissible choices of intervals $\left\{I_{j} ; 0 \leq j \leq k\right\}$.

Then we let $U_{1}(T)$ (resp. $U_{2}(T)$ ) be the completion of $c_{00}(T)$ under $\|\cdot\|_{1}$ (resp. $\|\cdot\|_{2}$ ). For any $A \subseteq T$, we denote by $U_{1}(A)$ (resp. $U_{2}(A)$ ) the closed 
subspace of $U_{1}(T)$ (resp. $\left.U_{2}(T)\right)$ generated by $\left\{\chi_{s} ; s \in A\right\}$. In this notation, the following holds.

TheOREM 1.2. Let $\theta \in \mathcal{T}$.

(i) If $\theta$ is not well founded, then $U_{1}(\theta)$ and $U_{2}(\theta)$ are isomorphic to $U$, thus universal.

(ii) If $\theta$ is well founded, then $U_{2}(\theta)$ is reflexive, and $U_{1}(\theta)$ has the Schur property, thus contains no infinite-dimensional reflexive space.

To prove Theorem 1.2, we need several technical lemmas, whose proofs are given later on.

LEMma 1.3. The sequence $\left(\chi_{s_{i}} ; i \in \omega\right)$ determines a basis for $U_{1}(T)$ and $U_{2}(T)$. For any $A \subseteq T,\left(\chi_{s_{i}} ; s_{i} \in A\right)$ determines a basis for $U_{1}(A)$ and $U_{2}(A)$.

Lemma 1.4. Let $b$ be a branch of $T$. Then

(i) The spaces $U_{1}(\{s ; s \prec b\}), U_{2}(\{s ; s \prec b\})$ and $U$ are isomorphic.

(ii) If $\theta \in \mathcal{T}$ and if $b$ is a branch of $\theta$, then for $r \in\{1,2\}, U_{r}(\{s ; s \prec b\})$ is a complemented subspace of $U_{r}(\theta)$.

Lemma 1.5. Let $\left(A_{i}\right)_{i \in \omega}$ be a sequence of subsets of $T$ such that every branch meets at most one of these subsets. Then for $r \in\{1,2\}$ the spaces $U_{r}\left(\bigcup_{i \in \omega} A_{i}\right)$ and $\left(\sum_{i \in \omega} \oplus U_{r}\left(A_{i}\right)\right)_{r}$ are isometric.

Lemma 1.6. Let $\left(X_{j}\right)_{j \in \omega}$ be a sequence of Banach spaces with the Schur property. Then $X=\left(\sum_{j \in \omega} \oplus X_{j}\right)_{1}$ has the Schur property.

Proof of Theorem 1.2. (i) If $\theta$ is not well founded, we pick a branch $b$ of $\theta$. By Lemmas 1.3 and 1.4, $U_{1}(\theta)$ and $U_{2}(\theta)$ are Banach spaces with a basis, which contain an isomorphic complemented copy of $U(b)=U$, and hence contain an isomorphic complemented copy of every Banach space with a basis. By Theorem 1.1, $U_{1}(\theta), U_{2}(\theta)$ and $U$ are isomorphic.

(ii) For $\theta \in \mathcal{T}, s \in T$ and $i \in \omega$, we define

$$
s \frown \theta=\{s \frown t ; t \in \theta\}, \quad \theta_{i}=\{t \in T ;(i) \frown t \in \theta\} .
$$

FACT. If $\theta$ is well founded, then for any $s \in T, U_{1}(s \frown \theta)$ has the Schur property, and $U_{2}(s \frown \theta)$ is reflexive.

With this fact, if $\theta$ is well founded, then $U_{1}(\theta)=U_{1}(\emptyset \frown \theta)$ has the Schur property, and $U_{2}(\theta)=U_{2}(\emptyset \frown \theta)$ is reflexive, thus (ii) is proved.

We show the fact by transfinite induction on the height ht $(\theta)$ of $\theta$.

Let $\alpha<\omega_{1}$. We assume that for every tree $\tau \in \mathcal{T}$ such that ht $(\tau)<\alpha$, $U_{1}(s \frown \tau)$ has the Schur property and $U_{2}(s \frown \tau)$ is reflexive for any $s \in T$. 
Let $\theta \in \mathcal{T}$ be such that ht $(\theta)=\alpha$, let $s \in T$ and $N_{s}=\{i \in \omega ; s \frown(i) \in \theta\}$. We let $A_{i}=s \frown(i) \frown \theta_{i}$ for $i \in N_{s}$, thus $\bigcup_{i \in N_{s}} A_{i}=s \frown(\theta \backslash\{s\})$ and every branch of $T$ meets at most one of the $A_{i}$ 's. If $i \in N_{s}$, then $\operatorname{ht}\left(\theta_{i}\right)<\alpha$, thus $U_{1}\left(A_{i}\right)=U_{1}\left(s \frown(i) \frown \theta_{i}\right)$ has the Schur property, and $U_{2}\left(A_{i}\right)=U_{2}\left(s \frown(i) \frown \theta_{i}\right)$ is reflexive.

By Lemma 1.5, for $r \in\{1,2\}$,

$$
U_{r}(s \frown(\theta \backslash\{s\}))=U_{r}\left(\bigcup_{i \in N_{s}} A_{i}\right)=\left(\sum_{i \in N_{s}} \oplus U_{r}\left(A_{i}\right)\right)_{r},
$$

thus $U_{2}(s \frown(\theta \backslash\{s\}))$ is reflexive, and by Lemma 1.6, $U_{1}(s \frown(\theta \backslash\{s\}))$ has the Schur property.

By Lemma 1.3, $\left(\chi_{s_{j}} ; j \in \omega, s_{j} \in s \frown \theta\right)$ is a basis of $U_{r}(s \frown \theta)$ with first element $\chi_{s}$ and the other elements generate $U_{r}(s \frown(\theta \backslash\{s\}))$. We have $U_{r}(s \frown \theta) \simeq \mathbb{R} \times U_{r}(s \frown(\theta \backslash\{s\}))$. Thus $U_{1}(s \frown \theta)$ has the Schur property and $U_{2}(s-\theta)$ is reflexive. The fact follows, and Theorem 1.2 is proved.

Now we have to show the four lemmas we used in the above proof.

Proof of Lemma 1.3. The proof is the same for $U_{1}(T)$ and $U_{2}(T)$. We give it for $U_{2}(T)$.

Let $\left(\lambda_{i}\right)_{i \in \omega}$ be a sequence in $\mathbb{R}, I$ be an interval of $T$, and $n, p \in \omega$. We denote by $c_{u}$ the basis constant of $\underline{u}$.

For $s \in T,\left(\sum_{i=0}^{n} \lambda_{i} \chi_{s_{i}}\right)(s)$ is equal to $\lambda_{\bar{s}}$ if $\bar{s} \leq n$, and 0 if not. Therefore

$$
\begin{aligned}
\left\|\sum_{s \in I}\left(\sum_{i=0}^{n} \lambda_{i} \chi_{s_{i}}\right)(s) u_{|s|}\right\| & =\left\|\sum_{\substack{s \in I \\
\bar{s} \leq n}} \lambda_{\bar{s}} u_{|s|}\right\| \leq c_{u}\left\|\sum_{\substack{s \in I \\
\bar{s} \leq n+p}} \lambda_{\bar{s}} u_{|s|}\right\| \\
& =c_{u}\left\|\sum_{s \in I}\left(\sum_{i=0}^{n+p} \lambda_{i} \chi_{s_{i}}\right)(s) u_{|s|}\right\|
\end{aligned}
$$

since if $s, s^{\prime} \in I$, then $s^{\prime} \succeq s$ iff $\bar{s}^{\prime} \geq \bar{s}$.

Let $\left\{I_{j} ; 0 \leq j \leq k\right\}$ be an admissible choice of intervals. We have

$$
\sum_{j=0}^{k}\left\|\sum_{s \in I_{j}}\left(\sum_{i=0}^{n} \lambda_{i} \chi_{s_{i}}\right)(s) u_{|s|}\right\|^{2} \leq c_{u}^{2} \sum_{j=0}^{k}\left\|\sum_{s \in I_{j}}\left(\sum_{i=0}^{n+p} \lambda_{i} \chi_{s_{i}}\right)(s) u_{|s|}\right\|^{2} .
$$

Thus $\left\|\sum_{i=0}^{n} \lambda_{i} \chi_{s_{i}}\right\|_{2} \leq c_{u}\left\|\sum_{i=0}^{n+p} \lambda_{i} \chi_{s_{i}}\right\|_{2}$ and $\left(\chi_{s_{i}}\right)_{i \in \omega}$ is a basic sequence. Lemma 1.3 follows.

Proof of Lemma 1.4. Let $r \in\{1,2\}$.

(i) We write $b_{j}=b_{\mid j}$ for $j \in \omega$. It is sufficient to prove that $\underline{u}$ and the basis $\left(\chi_{b_{j}} ; j \in \omega\right)$ of $U_{r}(\{s ; s<b\})$ are equivalent. Let $\left(\lambda_{j}\right)_{j=0}^{n} \in \mathbb{R}^{<\omega}$ and 
$y=\sum_{j=0}^{n} \lambda_{j} \chi_{b_{j}}$. As $b$ is a branch and $\left|b_{j}\right|=j$, we have

$$
\begin{aligned}
\|y\|_{r} & =\sup \left\{\left\|\sum_{s \in I} y(s) u_{|s|}\right\| ; I \text { interval, } I \subseteq\{s ; s \prec b\}\right\} \\
& =\sup \left\{\left\|\sum_{j=l}^{m} \lambda_{j} u_{j}\right\| ; 0 \leq l \leq m \leq n\right\}
\end{aligned}
$$

and with $c_{u}$ being the basis constant of $\underline{u}$,

$$
\left\|\sum_{j=0}^{n} \lambda_{j} u_{j}\right\| \leq\|y\|_{r} \leq 2 c_{u}\left\|\sum_{j=0}^{n} \lambda_{j} u_{j}\right\|
$$

Thus $\left(\chi_{b_{j}} ; j \in \omega\right)$ and $\underline{u}$ are equivalent, and (i) follows.

(ii) Let $y=\sum_{i \in \omega} y\left(s_{i}\right) \chi_{s_{i}}$ be an element of $U_{r}(\theta)$. We have

$$
\left\|\sum_{\substack{i \in \omega \\ s_{i} \in b}} y\left(s_{i}\right) \chi_{s_{i}}\right\|_{r}=\sup \left\{\left\|\sum_{s \in I} y(s) u_{|s|}\right\| ; I \text { interval, } I \subseteq\{s ; s \prec b\}\right\} \leq\|y\|_{r}
$$

and (ii) follows.

Proof of Lemma 1.5. The proof is the same for $r=1$ and $r=2$. We give it when $r=2$. Pick $y \in \operatorname{sp}\left(\left\{\chi_{s} ; s \in \operatorname{sp}\left(\chi_{s} ; s \in \bigcup_{i \in \omega} A_{i}\right\}\right)\right.$. We let $y_{i}=\sum_{s \in A_{i}} y(s) \chi_{s}$. The set $\left\{y_{i} ; i \in \omega, y_{i} \neq 0\right\}$ is finite, thus there is $m \in \omega$ such that $y=\sum_{i=0}^{m} y_{i}$. Clearly Lemma 1.5 follows from the following Fact.

FACT. $\|y\|_{2}^{2}=\sum_{i=0}^{m}\left\|y_{i}\right\|_{2}^{2}$.

Indeed, let $\left\{I_{j} ; 0 \leq j \leq k\right\}$ be an admissible choice of intervals. We set, for $0 \leq j \leq k$ and $0 \leq i \leq m, I_{j}(y)=\sum_{s \in I_{j}} y(s) u_{|s|}$ and $M_{i}=\{j \in \omega$; $\left.0 \leq j \leq k, I_{j} \cap A_{i} \neq \emptyset\right\}$. The largest interval with ends in $I_{j} \cap A_{i}$ is denoted by $J_{j}^{i}$. For any $i \in \omega,\left\{J_{j}^{i} ; j \in M_{i}\right\}$ is an admissible choice of intervals, thus

$$
\sum_{j=0}^{k}\left\|I_{j}(y)\right\|^{2}=\sum_{i=0}^{m} \sum_{j \in M_{i}}\left\|J_{j}^{i}\left(y_{i}\right)\right\|^{2} \leq \sum_{i=0}^{m}\left\|y_{i}\right\|_{2}^{2} .
$$

And it follows by taking the supremum over admissible choices of intervals that

$$
\|y\|_{2}^{2} \leq \sum_{i=0}^{m}\left\|y_{i}\right\|_{2}^{2}
$$

Now for any $i, 0 \leq i \leq m$, let $\left\{I_{j}^{i} ; 0 \leq j \leq k_{i}\right\}$ be an admissible choice of intervals. We denote by $\widetilde{I}_{j}^{i}$ the largest interval with ends in $I_{j}^{i} \cap A_{i}$. Then $\left\{\widetilde{I}_{j}^{i} ; 0 \leq i \leq m, 0 \leq j \leq k_{i}\right\}$ is an admissible choice of intervals, because every branch of $T$ meets at most one of the $A_{i}$ 's. For any $i$, we have successively 


$$
\begin{aligned}
& \sum_{j=0}^{k_{i}}\left\|I_{j}^{i}\left(y_{i}\right)\right\|^{2}=\sum_{j=0}^{k_{i}}\left\|\widetilde{I}_{j}^{i}\left(y_{i}\right)\right\|^{2}=\sum_{j=0}^{k_{i}}\left\|I_{j}^{i}(y)\right\|^{2}, \\
& \sum_{i=0}^{m} \sum_{j=0}^{k_{i}}\left\|I_{j}^{i}\left(y_{i}\right)\right\|^{2}=\sum_{i=0}^{m} \sum_{j=0}^{k_{i}}\left\|\widetilde{I}_{j}^{i}(y)\right\|^{2} \leq\|y\|_{2}^{2},
\end{aligned}
$$

thus

$$
\sum_{i=0}^{m}\left\|y_{i}\right\|_{2}^{2} \leq\|y\|_{2}^{2} .
$$

The fact follows, and Lemma 1.5 is proved.

Proof of Lemma 1.6. It is sufficient to show that if $\left(x_{n}\right)_{n \in \omega} \in X^{\omega}$ is weakly convergent to 0 , then it is norm-convergent. By contradiction, let $\left(x_{n}\right)_{n \in \omega} \in X^{\omega}$ be a sequence which is weakly convergent to 0 , and suppose it is not norm-convergent. We can suppose there is $\varepsilon>0$ such that $\left\|x_{n}\right\| \geq 2 \varepsilon$ for every $n$ (if not, take a subsequence). For $n \in \omega$, let $x_{n}=\sum_{j \in \omega} x_{n}^{j}$ with $x_{n}^{j} \in X_{j}$. For any $j,\left(x_{n}^{j}\right)_{n \in \omega}$ is weakly convergent to 0 since the natural projection from $X$ onto $X_{j}$ is weakly continuous. Thus $\left(x_{n}^{j}\right)_{n \in \omega}$ is normconvergent to 0 .

Using the "gliding hump" technique, we build by induction a sequence $\left(y_{m}\right)_{m \in \omega}$ in $X$ and strictly increasing sequences $\left(n_{m}\right)_{m \in \omega}$ and $\left(j_{m}\right)_{m \in \omega}$ in $\omega$ which satisfy

$$
\begin{gathered}
n_{0}=0, \quad j_{-1}=0<j_{0}, \quad y_{m}=\sum_{j=j_{m-1}}^{j_{m}-1} x_{n_{m}}^{j} \in \sum_{j=j_{m-1}}^{j_{m}-1} \oplus X_{j}=Z_{m}, \\
\left\|x_{n_{m}}-y_{m}\right\| \leq \frac{\varepsilon}{m+1}, \quad\left\|y_{m}\right\| \geq \varepsilon .
\end{gathered}
$$

Then $\left(y_{m}\right)_{m \in \omega}$ is weakly convergent to 0 , since $\left(x_{n_{m}}\right)_{m \in \omega}$ is and since $\left\|x_{n_{m}}-y_{m}\right\| \rightarrow 0$. But for any $m \in \omega$ there is $f_{m} \in Z_{m}^{\star}=\left(\sum_{j=j_{m-1}}^{j_{m}-1} \oplus X_{j}^{\star}\right)_{\infty}$ with norm 1 such that $f_{m}\left(y_{m}\right) \geq \varepsilon$. We consider the function $f \in X^{\star}$ defined by $f(y)=\sum_{m \in \omega} f_{m}\left(y_{\mid Z_{m}}\right)$ where $\left(y_{\mid Z_{m}}\right)$ is the image of $y$ under the natural projection on $Z_{m}$. Then the norm of $f$ is $1, f\left(y_{m}\right)=f_{m}\left(y_{m}\right) \geq \varepsilon$ for any $m \in \omega$, thus $\left(y_{m}\right)_{m \in \omega}$ cannot converge weakly to 0 , a contradiction.

Consequently, $\left(x_{n}\right)_{n \in \omega}$ is norm-convergent to 0 and Lemma 1.6 follows.

\section{Codings of separable Banach spaces up to isomorphism, ana-} lytic non-Borel relations. In this section, we use a natural representation of the collection of all the separable Banach spaces to make it into a set. We show that for natural codings by standard Borel spaces, the relations of linear isomorphism and being isomorphic to a subspace, quotient, direct sum lead to analytic non-Borel relations. Moreover the various codings we can choose are essentially equivalent. 
We denote by $\mathcal{S E}(Z)$ the subset of $\mathcal{F}(Z)$ consisting of the closed subspaces of a Banach space $Z$. The fact that $C(\Delta)$ is universal suggests coding separable Banach spaces by Banach subspaces of $C(\Delta)$. We abbreviate $\mathcal{S E}(C(\Delta))$ to $\mathcal{S E}$. If $X$ is a separable Banach space, we will denote by $\langle X\rangle$ the equivalence class $\{Y \in \mathcal{S E} ; Y \simeq X\}$ of the isomorphism relation $\simeq$. We now define our codings.

DeFINITION 2.1. A coding of separable Banach spaces up to isomorphism is a map from a set $E$ onto the quotient set $\mathcal{S E} / \simeq$. The canonical coding is the quotient map from $\mathcal{S E}$ onto $\mathcal{S E} / \simeq$ which we denote by $c$.

The following proposition shows in particular that the set $\mathcal{S E}$ is a standard Borel space.

Proposition 2.2. Let $Z$ be a separable Banach space. Then $\mathcal{S E}(Z)$ is a Borel subset of $\mathcal{F}(Z)$ equipped with the Effros-Borel structure.

Proof. We have

$$
\mathcal{S E}(Z)=\{F \in \mathcal{F}(Z) ; F \text { satisfies }(\mathrm{a})\} \cap\{F \in \mathcal{F}(Z) ; F \text { satisfies }(\mathrm{b})\}
$$

with

$$
\begin{aligned}
& \text { (a) }: \forall \lambda \in \mathbb{R}, x \in F \Rightarrow \lambda x \in F, \\
& \text { (b) }:(x, y) \in F^{2} \Rightarrow x+y \in F .
\end{aligned}
$$

Let $\mathcal{O}$ be a countable basis of open subsets of $Z$. When $O, O^{\prime} \in \mathcal{O}$ and $\lambda \in \mathbb{R}^{\star}$, the subsets

$$
\begin{aligned}
\lambda O & =\{x \in Z ; \exists y \in O, y=\lambda x\} \quad \text { and } \\
O+O^{\prime} & =\left\{x \in Z ; \exists y \in O, \exists y^{\prime} \in O^{\prime}, y+y^{\prime}=x\right\}
\end{aligned}
$$

are open.

We leave to the reader the easy verification of the following fact.

FACT. Let $F \in \mathcal{F}(Z)$. We have the equivalences:

(a) $\Leftrightarrow\left(\mathrm{a}^{\prime}\right): \forall \lambda \in \mathbb{Q}^{\star}, \forall O \in \mathcal{O}, O \cap F \neq \emptyset \Rightarrow \lambda O \cap F \neq \emptyset$

$$
\text { (b) } \left.\Leftrightarrow\left(\mathrm{b}^{\prime}\right): \forall O \in \mathcal{O}, \forall O^{\prime} \in \mathcal{O}, \begin{array}{l}
O \cap F \neq \emptyset \\
O^{\prime} \cap F \neq \emptyset
\end{array}\right\} \Rightarrow\left(O+O^{\prime}\right) \cap F \neq \emptyset .
$$

Consequently, in $\mathcal{F}(Z)$ we have

$$
\{F ; F \text { satisfies (a) }\}=\bigcap_{\lambda \in \mathbb{Q}^{\star}} \bigcap_{O \in \mathcal{O}}(\{F ; \lambda O \cap F \neq \emptyset\} \cup\{F ; O \cap F=\emptyset\}),
$$

$\{F ; F$ satisfies (b) $\}$

$$
=\bigcup_{\left(O, O^{\prime}\right) \in \mathcal{O}^{2}}\left(\left\{F ;\left(O+O^{\prime}\right) \cap F \neq \emptyset\right\} \cup\{F ; O \cap F=\emptyset\} \cup\left\{F ; O^{\prime} \cap F=\emptyset\right\}\right) \text {. }
$$

Thus these two subsets are Borel, and $\mathcal{S E}(Z)$ is Borel as well. 
The main result of this section is the following theorem.

THEOREM 2.3. (i) The isomorphism relation $\simeq$ is analytic non-Borel in $\mathcal{S E}^{2}$ and it has no analytic section. In fact, the equivalence class $\langle U\rangle$ is not Borel.

(ii) The relations $\mathcal{S}=\{X, Y ; X \subset Y\}, \mathcal{Q}=\{(X, Y) ; Y \Rightarrow X\}$ and $\mathcal{C}=\{(X, Z) ; \exists Y \in \mathcal{S E}, Z \simeq X \oplus Y\}$ are analytic non-Borel in $\mathcal{S E}^{2}$. The relation $\mathcal{D}=\{(X, Y, Z) ; Z \simeq X \oplus Y\}$ is analytic non-Borel in $\mathcal{S E}^{3}$.

The assertion (i) means that isomorphism cannot be defined in a Borel way if we use the canonical coding. By Proposition 2.8 we will see that this remains true if we replace the canonical coding by other natural codings of separable Banach spaces.

Theorem 2.3 is clearly a consequence of Propositions 2.5 and 2.7 below. We can consider that $U_{2}(T)$ is a subspace of $C(\Delta)$. Thus $U_{2}(\theta) \in \mathcal{S E}$ for any $\theta \in \mathcal{T}$. We need the following simple lemma.

Lemma 2.4. The map $\varphi: \mathcal{T} \rightarrow \mathcal{S E}$ defined by $\varphi(\theta)=U_{2}(\theta)$ is Borel.

Proof. Let $O$ be an open subset of $C(\Delta)$. It is sufficient to prove that $\Omega=\left\{\theta \in \mathcal{T} ; U_{2}(\theta) \cap O \neq \emptyset\right\}$ is Borel. Since $\left(\chi_{s_{i}} ; i \in \omega, s_{i} \in \theta\right)$ defines a basis of $U_{2}(\theta)$, we have the equivalence: $U_{2}(\theta) \cap O \neq \emptyset$ iff there is some $\underline{\lambda}=\left(\lambda_{i}\right)_{i=0}^{n} \in \mathbb{Q}^{<\omega}$ such that $\sum_{i=0}^{n} \lambda_{i} \chi_{s_{i}} \in O$ and if $\lambda_{i} \neq 0$ then $s_{i} \in \theta$.

Let $\Lambda=\left\{\underline{\lambda} \in \mathbb{Q}^{<\omega} ; \sum_{i} \lambda_{i} s_{i} \in O\right\}$ and for $\underline{\lambda} \in \mathbb{Q}^{<\omega}$ set $\operatorname{supp}(\underline{\lambda})=$ $\left\{i \in \omega ; \lambda_{i} \neq 0\right\}$. Then

$$
\Omega=\bigcup_{\underline{\lambda} \in \Lambda} \bigcap_{i \in \operatorname{supp}(\underline{\lambda})}\left\{\theta \in \mathcal{T} ; s_{i} \in \theta\right\}
$$

thus $\Omega$ is Borel since $\left\{\theta \in \mathcal{T} ; s_{i} \in \theta\right\}$ is an open and closed subset.

Proposition 2.5. The class $\langle U\rangle$ is not Borel and the relations $\simeq, \mathcal{S}$, $\mathcal{Q}, \mathcal{C}$ and $\mathcal{D}$ are not Borel.

Proof. Since $\varphi^{-1}(\langle U\rangle)=\mathrm{MF}$ and MF is not Borel, it follows that $\langle U\rangle$ is not a Borel class, and consequently $\simeq$ is not a Borel relation.

By Lemma 2.4, the maps

$$
\begin{aligned}
& \varphi_{1}: \mathcal{T} \rightarrow(\mathcal{S E})^{2} \quad \text { defined by } \quad \varphi_{1}(\theta)=\left(U, U_{2}(\theta)\right) \\
& \text { and } \varphi_{2}: \mathcal{T} \rightarrow(\mathcal{S E})^{3} \quad \text { defined by } \varphi_{2}(\theta)=\left(U,\{0\}, U_{2}(\theta)\right)
\end{aligned}
$$

are Borel. Moreover it is easy to check that

$$
\varphi_{1}^{-1}(\mathcal{S})=\varphi_{1}^{-1}(\mathcal{Q})=\varphi_{1}^{-1}(\mathcal{C})=\varphi_{2}^{-1}(\mathcal{D})=\mathrm{MF},
$$

since $U_{2}(\theta)$ is reflexive if $\theta \in \mathrm{WF}$ and $U_{2}(\theta) \simeq U$ if $\theta \in \mathrm{MF}$ by Theorem 1.2. Therefore since MF is not Borel, we conclude that $\mathcal{S}, \mathcal{Q}, \mathcal{C}$ and $\mathcal{D}$ are not Borel sets. 
The following lemma, whose easy proof is left to the reader, is useful to show that $\simeq, \mathcal{S}, \mathcal{Q}, \mathcal{C}$ and $\mathcal{D}$ are analytic.

Lemma 2.6. Let $P$ be a Polish space and $Z$ be a separable Banach space.

(i) $\{(F, y) ; y \in F\}$ is Borel in $\mathcal{F}(P) \times P$, and consequently $\{(Y, y)$; $y \in Y\}$ is Borel in $\mathcal{S E}(Z) \times Z$.

(ii) $\{(Y, y) ; \overline{\mathrm{sp}}(y)=Y\}$ is Borel in $\mathcal{S E}(Z) \times Z^{\omega}$.

(iii) $\{(\underline{x}, \underline{y}) ; \underline{x} \sim \underline{y}\}$ is Borel in $Z^{\omega} \times Z^{\omega}$.

(iv) $\{(F, G) ; G \subseteq F\}$ is Borel in $\mathcal{F}(P)^{2}$, and consequently $\{(X, Y)$; $Y \subseteq X\}$ is Borel in $\mathcal{S E}(Z)^{2}$.

Proposition 2.7. (i) The isomorphism relation $\simeq$ is analytic in $\mathcal{S E}^{2}$ and has no analytic section.

(ii) The relations $\mathcal{S}, \mathcal{Q}, \mathcal{C}$ in $\mathcal{S E}^{2}$ and $\mathcal{D}$ in $\mathcal{S E}^{3}$ are analytic.

Proof. (i) First it is easy to verify the following.

FACT. Let $X, Y$ be two separable Banach spaces. Then $X \simeq Y$ iff there are some $\underline{x} \in X^{\omega}$ and $\underline{y} \in Y^{\omega}$ such that $\underline{x} \sim \underline{y}, \overline{\mathrm{sp}}(\underline{x})=X$ and $\overline{\mathrm{sp}}(\underline{y})=Y$.

By Lemma 2.6(ii), (iii), the subset $\{(X, Y, \underline{x}, y) ; \overline{\operatorname{sp}}(\underline{x})=X, \overline{\mathrm{sp}}(\underline{y})=Y$, $\underline{x} \sim y\}$ in $\mathcal{S E}^{2} \times\left(C(\Delta)^{\omega}\right)^{2}$ is Borel. The image of this set under the natural projection onto $\mathcal{S E}^{2}$ is analytic, and by the Fact, this image is $\{(X, Y)$; $X \simeq Y\}$. Thus $\simeq$ is analytic. Then the class $\langle U\rangle$ is analytic, and non-Borel by Proposition 2.5 .

It remains to prove that the relation $\simeq$ in $\mathcal{S E}$ has no analytic section. Working by contradiction, we assume that $\Sigma$ is an analytic section, and $U^{\prime} \in \Sigma$ is such that $U^{\prime} \simeq U$. Then $\Sigma \backslash\left\{U^{\prime}\right\}$ is analytic. We consider the maps $\pi_{1}, \pi_{2}: \mathcal{S} \mathcal{E}^{2} \rightarrow \mathcal{S E}$ defined by $\pi_{1}(X, Y)=X$ and $\pi_{2}(X, Y)=Y$. The subset $\left\{(X, Y) ; Y \in \Sigma \backslash\left\{U^{\prime}\right\}, X \simeq Y\right\}=\{(X, Y) ; Y \in \Sigma, X \simeq Y, X \notin\langle U\rangle\}$ is analytic. The $\pi_{1}$-image of this last set is $\{X ; X \notin\langle U\rangle\}$ since $\Sigma$ is a section, and this image is analytic. Thus its complement $\langle U\rangle$ is coanalytic. By the separation theorem, $\langle U\rangle$ is Borel, a contradiction. Hence the relation $\simeq$ has no analytic section.

(ii) We give the main ideas of the proof; the details are left to the reader.

Since $\mathcal{S}=\{(X, Y) ; \exists Z \in \mathcal{S E}, Z \subseteq Y, X \simeq Z\}$, by (i) and Lemma 2.6(iv), $\mathcal{S}$ is analytic.

To prove that $\mathcal{Q}$ is analytic, we use the following easy result and Lemma 2.6.

FACT. In $\mathcal{S E}^{2},(X, Y) \in \mathcal{Q}$ iff there are $Z \in \mathcal{S E}$ and $\underline{x}, \underline{y}, \underline{z} \in C(\Delta)^{\omega}$ such that

(1) $\overline{\mathrm{sp}}(\underline{x})=X, \overline{\mathrm{sp}}(\underline{y})=Y, \overline{\mathrm{sp}}(\underline{z})=Z, Z \subseteq Y$,

(2) $\underline{x} \sim \underline{y}^{\prime}$, where $\underline{y}^{\prime}$ is the image of $\underline{y}$ under the quotient map from $Y$ onto $Y / Z$. 
Finally, to prove that $\mathcal{C}$ and $\mathcal{D}$ are analytic, we use the following equivalence.

FACT. Let $(X, Y, Z) \in \mathcal{S E}^{3}$. Then $Z \simeq X \oplus Y$ if and only if there is some $(\underline{x}, \underline{y}, \underline{z}) \in\left(C(\Delta)^{\omega}\right)^{3}$ satisfying the following two conditions:

(3) $\overline{\mathrm{sp}}(\underline{x})=X, \overline{\mathrm{sp}}(\underline{y})=Y, \overline{\mathrm{sp}}(\underline{z})=Z,\left(z_{2 i}\right)_{i \in \omega} \sim \underline{x},\left(z_{2 i+1}\right)_{i \in \omega} \sim \underline{y}$,

(4) there is a linear continuous projection $\pi$ from $\overline{\mathrm{sp}}(\underline{x} \cup y)$ onto $\overline{\mathrm{sp}}(\underline{x})$ such that $\operatorname{ker} \pi=\overline{\mathrm{sp}}(\underline{y})$.

We now consider other natural codings of separable Banach spaces up to isomorphism. Our goal is to show that they lead to the same estimates on the complexity of the relevant sets. The following maps $c_{a}, c_{b}$ and $c_{d}$ are codings, in the sense of Definition 2.1, since $C(\Delta)$ is universal and since every separable Banach space is isometric to a quotient space of $\ell_{1}(\omega)$ :

$$
\begin{array}{rlrl}
c_{a} & : \mathcal{S E}\left(\ell_{1}(\omega)\right) \rightarrow \mathcal{S E} / \simeq, & c_{a}(W) & =\left\langle\ell_{1}(\omega) / W\right\rangle, \\
c_{b}: C(\Delta)^{\omega} \rightarrow \mathcal{S E} / \simeq, & c_{b}(\underline{v}) & =\langle\overline{\mathrm{sp}}(\underline{v})\rangle, \\
c_{d}: \ell_{1}(\omega)^{\omega} \rightarrow \mathcal{S E} / \simeq, & c_{d}(\underline{w}) & =\left\langle\ell_{1}(\omega) / \overline{\mathrm{sp}}(\underline{w})\right\rangle .
\end{array}
$$

We will show that these codings lead to identical results as in Theorem 2.3. This relies on the following general statement.

Proposition 2.8. Let $F$ and $G$ be two standard Borel spaces, $E$ be a set, $c_{1}: F \rightarrow E$ and $c_{2}: G \rightarrow E$ be two surjections. Assume that the set $\left\{(f, g) ; c_{1}(f)=c_{2}(g)\right\}$ is analytic in $F \times G$.

(i) If $A \subseteq F$ is analytic, then $c_{2}^{-1}\left(c_{1}(A)\right)$ is analytic as well.

(ii) Let $C \subseteq E$. Then $c_{1}^{-1}(C)$ is analytic (resp. coanalytic) in $F$ iff $c_{2}^{-1}(C)$ is analytic (resp. coanalytic) in $G$.

In particular this is true when $c_{1}=c$ and $c_{2}: G \rightarrow \mathcal{S E} / \simeq$ is a coding of separable Banach spaces up to isomorphism from a standard Borel space $G$ such that $\left\{(X, g) \in \mathcal{S E} \times G ; c(x)=c_{2}(g)\right\}$ is analytic. We leave it to the reader to verify that if $c_{2} \in\left\{c_{a}, c_{b}, c_{d}\right\}$, then this condition is fulfilled. Thus if we replace $c$ by $c_{a}, c_{b}$ or $c_{d}$, we obtain the same results as in Theorem 2.3. Note that by the separation theorem, assertion (ii) of Proposition 2.8 also holds when "analytic" is replaced by "Borel".

Proof of Proposition 2.8. (i) Let $A$ be an analytic subset of $F$. We have the equivalence

$$
g \in c_{2}^{-1}\left(c_{1}(A)\right) \Leftrightarrow \exists f \in A, c_{1}(f)=c_{2}(g) .
$$

From this, we easily deduce that $c_{2}^{-1}\left(c_{1}(A)\right)$ is analytic.

(ii) Let $C \subseteq E$. Assume $c_{1}^{-1}(C)$ is analytic. Since $c_{1}$ is a surjection, $c_{1}\left(c_{1}^{-1}(C)\right)=C$, thus by (i), $c_{2}^{-1}(C)=c_{2}^{-1}\left[c_{1}\left(c_{1}^{-1}(C)\right)\right]$ is analytic. 
Now if $c_{1}^{-1}(C)$ is coanalytic, then, with $D=E \backslash C, c_{1}^{-1}(D)=F \backslash c_{1}^{-1}(C)$ is analytic. Thus $c_{2}^{-1}(D)$ is analytic as well, and its complement $c_{2}^{-1}(C)$ is coanalytic. This finishes the proof.

We conclude this section with an open question. Using Kwapień's theorem $([\mathrm{Kw}])$, it is not difficult to see that the class $\left\langle\ell_{2}(\omega)\right\rangle$ is Borel. Indeed by Kwapien's theorem an infinite-dimensional separable Banach space which is of type 2 and cotype 2 is isomorphic to $\ell_{2}(\omega)$. And "being of type 2 " and "being of cotype 2" are Borel conditions. Using the coding $c_{b}$, we prove this for type 2 .

A separable Banach space $X$ is of type 2 if there is some $M \in \mathbb{R}$ such that for any finite sequence $\left(x_{j}\right)_{j=0}^{n}$ in $X$ we have

$$
\frac{1}{2^{n}} \sum_{\varepsilon_{j}= \pm 1}\left\|\sum_{j=0}^{n} \varepsilon_{j} x_{j}\right\| \leq M\left(\sum_{j=0}^{n}\left\|x_{j}\right\|^{2}\right)^{1 / 2} .
$$

We easily deduce: for any $\underline{v} \in C(\Delta)^{\omega}$, the space $\overline{\mathrm{sp}}(\underline{v})$ is of type 2 if and only if there is some $M \in \mathbb{Q}^{+}$such that for any $n \in \omega$ and any $\left(\underline{\lambda}^{j}\right)_{j=0}^{n} \in$ $\left(\mathbb{Q}^{<\omega}\right)^{n+1}$, we have

$$
\frac{1}{2^{n}} \sum_{\varepsilon_{j}= \pm 1}\left\|\sum_{j=0}^{n} \varepsilon_{j} \underline{\lambda}^{j} \cdot \underline{v}\right\| \leq M\left(\sum_{j=0}^{n}\left\|\underline{\lambda}^{j} \cdot \underline{v}\right\|^{2}\right)^{1 / 2}
$$

where $\underline{\lambda}^{j} \cdot \underline{v}=\sum_{i} \lambda_{i}^{j} v_{i}$. Consequently, $\left\{\bar{v} \in C(\Delta)^{\omega} ; \overline{\operatorname{sp}}(\underline{v})\right.$ is of type 2$\}$ is Borel.

For cotype 2, the proof is similar, since by definition a separable Banach space $X$ is of cotype 2 if there is some $M \in \mathbb{R}^{*+}$ such that for any finite sequence $\left(x_{j}\right)_{j=0}^{n}$ in $X$ we have

$$
\frac{1}{2^{n}} \sum_{\varepsilon_{j}= \pm 1}\left\|\sum_{j=0}^{n} \varepsilon_{j} x_{j}\right\| \geq \frac{1}{M}\left(\sum_{j=0}^{n}\left\|x_{j}\right\|^{2}\right)^{1 / 2} .
$$

Consequently, $\left\langle\ell_{2}(\omega)\right\rangle$ is Borel.

It follows from Bourgain's work ([Bou4]) that the equivalence classes $\left\langle L_{p}(0,1)\right\rangle$ when $1<p<\infty$ and $p \neq 2$ are not Borel. Thus a natural question is:

Problem 2.9. Is there some separable Banach space $X$ such that $X$ is not isomorphic to $\ell_{2}(\omega)$ and its isomorphism class $\langle X\rangle$ is Borel?

\section{Topological complexity of families of separable Banach spaces} which are stable under isomorphism. We identify a family of separable Banach spaces which is stable under isomorphism with a subset of $\mathcal{S E} / \simeq$. Referring to the canonical coding $c$, it is natural to define the topological complexity of such a family as follows. 
Definition 3.1. A family $\mathcal{G} \subseteq \mathcal{S E} / \simeq$ is analytic (resp. coanalytic, Borel) if $c^{-1}(\mathcal{G})$ is analytic (resp. coanalytic, Borel).

The following theorem, which is the main result of this section, is an extension of a seminal result of J. Bourgain ([Bou3]) which appears here as a corollary (Corollary 3.4(i)).

TheOREm 3.2. Let $\mathcal{A}$ be an analytic family of separable Banach spaces, stable under isomorphism, which contains all separable reflexive spaces. Then $\mathcal{A}$ contains a space which is universal for all separable Banach spaces.

An informal consequence of Theorem 3.2 is that any hereditary property which is "analytic" (that is, whose statement starts with "there exists"...) is true for every separable Banach space if it is true for all reflexive Banach spaces.

Proof. The map $\varphi$ is defined in Lemma 2.4. If $\theta \in \mathrm{WF}$, then $\varphi(\theta)=U_{2}(\theta)$ is reflexive (Theorem 1.2), thus $\varphi^{-1}\left[c^{-1}(\mathcal{A})\right]$ is analytic and contains WF. Since WF is not analytic, there is some $\theta_{0} \in \operatorname{MF} \cap \varphi^{-1}\left[c^{-1}(\mathcal{A})\right]$, and $\varphi\left(\theta_{0}\right)=$ $U_{2}\left(\theta_{0}\right)$ is an element of $c^{-1}(\mathcal{A})$ which is isomorphic to $U$. Theorem 3.2 follows.

As a corollary, we obtain the topological complexity of some families of separable Banach spaces.

COROLlary 3.3. The following families of separable Banach spaces which are stable under isomorphism are coanalytic and not Borel:

(i) the family $\mathcal{G}_{r}$ of reflexive spaces,

(ii) the family $\mathcal{G}_{s}$ of spaces with separable dual,

(iii) the family $\mathcal{G}_{\ell}$ of spaces which do not contain an isomorphic copy of $\ell_{1}(\omega)$,

(iv) the family $\mathcal{G}_{c}$ of spaces which do not contain a complemented copy of $\ell_{1}(\omega)$,

(v) the family $\mathcal{G}_{n}$ of non-universal spaces,

(vi) the family $\mathcal{G}_{Z}$ of spaces which do not contain an isomorphic copy of the infinite-dimensional separable Banach space $Z$,

(vii) the family $\mathcal{G}_{p}$ of spaces with the Radon-Nikodym property. In fact, $c^{-1}(\mathcal{G})$ is a complete coanalytic set when $\mathcal{G}$ is one of these families.

This statement asserts that any characterization of the families (i) to (vii) will be at least as complex as the definition. Note that (iii) and (v) are special cases of (vi). We singled them out because of their particular importance.

Proof. Let $\mathcal{G}$ be one of these families, except the family $\mathcal{G}_{Z}$ when $Z$ is reflexive. Then $\mathcal{G}$ contains all the reflexive separable spaces, and does not 
contain a universal space (for (iv), note that $U$ contains a complemented copy of $\ell_{1}(\omega)$, and see the proof of Theorem 3.2). By Theorem 3.2, $\mathcal{G}$ is not analytic, thus not Borel. In fact, if $\theta \in \mathcal{T}$, then $\varphi(\theta) \in c^{-1}(\mathcal{G})$ iff $\theta \in \mathrm{WF}$. Hence by Lemma $2.4, c^{-1}(\mathcal{G})$ is a complete coanalytic subset if it is coanalytic.

Now if $Z$ is reflexive, we use the map $\Phi: \mathcal{T} \rightarrow \mathcal{S E}$ defined by $\Phi(\theta)=U_{1}(\theta)$ (see Section 1). Following similar lines to those in Lemma 2.4, it is not difficult to prove that $\Phi$ is Borel. By Theorem 1.2, if $\theta \in \mathrm{WF}$ then $\Phi(\theta)$ does not contain an isomorphic copy of $Z$, and if $\theta \notin \mathrm{WF}$ then $\Phi(\theta)$ contains an isomorphic copy of $Z$ since it is universal. Hence $c^{-1}\left(\mathcal{G}_{Z}\right)$ is a complete coanalytic subset if it is coanalytic, and is not Borel.

It remains to prove that all these families are coanalytic. If $\mathcal{G}$ is such a family, it is sufficient to show that the complement of $c^{-1}(\mathcal{G})$ is analytic. We give the main ideas for this proof, the details are left to the reader and we refer to [Bos] for a complete proof. In the following, $X$ is a separable Banach space.

To prove that $\mathcal{G}_{s}$ is coanalytic, we use the following equivalence due to I. Namioka and R. Phelps ([N-P] or [D-G-Z], Theorem I.5.2): $X^{\star}$ is not separable iff there is some $\varepsilon>0$ and some weak ${ }^{\star}$-compact $K \subseteq B_{X^{\star}}$ such that every subset $H \cap K \neq \emptyset$, with $H$ a $w^{\star}$-open half space, has a norm-diameter more than $\varepsilon$. Using the coding $c_{a}$, we prove that $c_{a}^{-1}\left(\mathcal{G}_{s}\right)=$ $\left\{W \in \mathcal{S E}\left(\ell_{1}(\omega)\right) ;\left(\ell_{1}(\omega) / W\right)^{\star}\right.$ separable $\}$ is coanalytic. Indeed if $W \in$ $\mathcal{S E}\left(\ell_{1}(\omega)\right)$, it is classical that $\left(\ell_{1}(\omega) / W\right)^{\star}$ and $W^{\perp}$ are isometric and $w^{\star}$ isomorphic, when $W^{\perp}$ is equipped with the topology inherited from the weak ${ }^{\star}$-topology of $\ell_{\infty}(\omega)$. Thus $\left(\ell_{1}(\omega) / W\right)^{\star}$ is not separable iff there is some $\varepsilon>0$ and some weak ${ }^{\star}$-closed subset $K$ of $B_{W \perp}$ such that every subset $H \cap K \neq \emptyset, H$ a $w^{\star}$-open half space, has a norm-diameter more than $\varepsilon$. Now it is easy to prove that $c_{a}^{-1}\left(\mathcal{G}_{s}\right)$ is coanalytic, and then by Proposition 2.8(ii), $\mathcal{G}_{s}$ is coanalytic.

To prove that $\mathcal{G}_{\ell}, \mathcal{G}_{n}$ and $\mathcal{G}_{Z}$ are coanalytic, we use the fact which follows easily from Theorem 2.3 , that the subset $\{X ; Z \subset X\}$ of $\mathcal{S E}$ is analytic, and hence $\left\{X ; \ell_{1}(\omega) \subset X\right\}$ and $\{X ; C(\Delta) \subset X\}$ are analytic as well.

In the same way, it is not difficult to prove that $\mathcal{G}_{c}$ is coanalytic.

And to prove that $\mathcal{G}_{p}$ is coanalytic, we use the equivalence: $X$ has the Radon-Nikodym property iff for any closed subset $F$ of $B_{X}$ and any $\varepsilon>0$, there is some $x \in F$ such that $x \notin \overline{\operatorname{conv}}(F \backslash B(x, \varepsilon))$. By classical methods we deduce that $\mathcal{G}_{p}$ is coanalytic. The verification is easy but tedious, and for more details we refer the reader to [Bos].

From Theorems 3.2 and 2.3 we obtain the following result, whose first assertion is Bourgain's result ([Bou2]). 
Corollary 3.4. Let $X$ be a separable Banach space.

(i) Every reflexive separable space has an isomorphic copy in $X$ iff $X$ is universal.

(ii) Every reflexive separable space is isomorphic to a subspace of a quotient space of $X$ iff $X$ contains an isomorphic copy of $\ell_{1}(\omega)$.

(iii) Every reflexive separable space is isomorphic to a quotient space of $X$ iff $X$ contains an isomorphic complemented copy of $\ell_{1}(\omega)$.

Proof. We can suppose $X \in \mathcal{S E}$. Let $\mathcal{A}_{1}$ be the family of Banach spaces which have an isomorphic copy in $X, \mathcal{A}_{2}$ be the family of spaces which are isomorphic to a subspace of a quotient space of $X$, and $\mathcal{A}_{3}$ be the family of spaces which are isomorphic to a quotient of $X$. These families are stable under isomorphism, and it is easy to see from Theorem 2.3 that they are analytic.

If every separable reflexive space is in $\mathcal{A}_{1}$, then by Theorem 3.2 a universal space is in $\mathcal{A}_{1}$, thus $X$ is universal. Since the other direction is clear, (i) follows.

Now if every separable reflexive space is in $\mathcal{A}_{2}$, then $U$ is in $\mathcal{A}_{2}$ by Theorem 3.2, thus $U$ is isomorphic to a subspace of a quotient of $X$. Since a subspace of a quotient of $X$ is isomorphic to a quotient of a subspace of $X$ (see for instance [L-T1], p. 85), there is some subspace $Z$ of $X$ such that $U$ is isomorphic to a quotient of $Z$. Then $\ell_{1}(\omega)$ is isomorphic to a complemented subspace of $U$ by a property of $U$ (Theorem 1.1), thus isomorphic to a quotient of $U$, and finally isomorphic to a quotient of $Z$. Hence it is easy to verify that $\ell_{1}(\omega)$ is isomorphic to a (complemented) subspace of $Z$, thus isomorphic to a subspace of $X$. The other direction is clear and (ii) follows.

In the same way, if every separable reflexive space is in $\mathcal{A}_{3}$, then $U$ is in $\mathcal{A}_{3}$, thus $U$, and $\ell_{1}(\omega)$, are isomorphic to a quotient of $X$. And $\ell_{1}(\omega)$ is isomorphic to a complemented subspace of $X$. The other direction is clear, (iii) follows, and the proof is complete.

The following corollary gives a characterization of $U$.

Corollary 3.5. Let $X$ be a separable Banach space with a basis. Then $X$ contains a complemented copy of every separable reflexive Banach space with a basis if and only if $X$ is isomorphic to $U$.

Proof. We suppose $X \in \mathcal{S E}$ satisfies the first assertion. The set $A=$ $\{Y \in \mathcal{S E} ; \exists Z \in \mathcal{S E}, X \simeq Y \oplus Z\}$ is analytic (Theorem 2.3). Thus $\varphi^{-1}(A)$ is analytic by Lemma 2.4, and contains WF (Theorem 1.2 and Lemma 1.4). Since WF is not analytic, there is some $\theta \notin \mathrm{WF}$ such that $U_{2}(\theta)$, which is isomorphic to $U$, is in $A$. Then $X$ contains a complemented copy of $U$, thus contains a complemented copy of every Banach space with a basis. By Theorem 1.1, $X$ is isomorphic to $U$. The other direction is clear. 
By Theorem 2.3, the set of spaces which are isomorphic to a subspace of a given space is analytic, hence by assertion (vi) of Corollary 3.3, the set of spaces which do not contain an isomorphic copy of $Z$ has no universal element. This can be formulated as follows.

Corollary 3.6 Let $X$ and $Z$ be infinite-dimensional separable Banach spaces such that $Z$ is not isomorphic to a subspace of $X$. Then there exists a separable Banach space $Y$ such that $Z$ is not isomorphic to a subspace of $Y$ and $Y$ is not isomorphic to a subspace of $X$.

In the following theorem, we determine the topological complexity of the family $\mathcal{J}$ consisting of the separable Banach spaces which do not contain an isomorphic copy of $\ell_{1}(\omega)$ and whose dual space is not separable. Quite naturally, this family brings us to the next level of complexity.

TheOREm 3.7. The family $\mathcal{J}$ is the difference of two coanalytic families, and is neither coanalytic nor analytic. In fact, $c^{-1}(\mathcal{J})$ reduces every difference of two coanalytic sets.

Proof. Since $\mathcal{J}=\mathcal{G}_{\ell} \backslash \mathcal{G}_{s}$, by Corollary $3.3, \mathcal{J}$ is the difference of two coanalytic sets.

Since WF is a complete coanalytic set, the set $\mathcal{D}_{0}=\mathrm{WF} \times \mathrm{MF}$ is neither analytic nor coanalytic, and reduces every difference of two coanalytic subsets; i.e. for any set $\mathcal{A}$ which is the difference of two coanalytic subsets in a Polish space $P$, there is some Borel map $\phi$ from $P$ to $\mathcal{S E}$ such that $\phi^{-1}\left(\mathcal{D}_{0}\right)=A$.

To prove Theorem 3.7, it is therefore sufficient to prove the following lemma.

Lemma 3.8. There is a Borel map $\varphi_{3}: \mathcal{T}^{2} \rightarrow \mathcal{S E}$ such that

$$
\varphi_{3}^{-1}\left(c^{-1}(\mathcal{J})\right)=\mathrm{WF} \times \mathrm{MF} .
$$

We first produce a family $\left\{J\left(A_{\theta}\right) ; \theta \in \mathcal{T}\right\}$ of separable Banach spaces such that $J\left(A_{\theta}\right)$ belongs to the family $\mathcal{J}$ iff $\theta \notin \mathrm{WF}$. As in Section 1 , we follow the lines of the construction of the James tree space, one of the first examples of Banach spaces in $\mathcal{J}$, built by R. C. James ([J1], or see [L-S]).

We equip the space $c_{00}(T)$ of all finitely supported functions from $T=$ $\omega^{<\omega}$ to $\mathbb{R}$ with the norm $\|\cdot\|_{J}$ defined by

$$
\|x\|_{J}^{2}=\sup \left\{\sum_{j=0}^{k}\left(\sum_{s \in I_{j}} x(s)\right)^{2}\right\}
$$

where the supremum is taken over $k \in \omega^{\star}$ and over the finite sets $\left\{I_{j} ; 0 \leq j \leq k\right\}$ of pairwise disjoint intervals. Then we let $J(T)$ be the completion of $c_{00}(T)$ under $\|\cdot\|_{J}$. For any $A \subseteq T$, we denote by $J(A)$ the 
closed subspace of $J(T)$ generated by $\left\{\chi_{s} ; s \in A\right\}$, where $\chi_{s}$ is the characteristic function of $\{s\}$. For instance, $J\left(\{0,1\}^{<\omega}\right)$ is the James tree space.

FACT 3.9. (i) $J(T)$ does not contain an isomorphic copy of $\ell_{1}(\omega)$

(ii) If $\theta \in \mathrm{WF}$, then $J(\theta)$ is reflexive.

Using an injection $\gamma$ from $\omega^{<\omega}$ to $\{0,1\}^{<\omega}$ such that if $s \prec t$ then $\gamma(s) \prec$ $\gamma(t)$, it is not difficult to see that $J(T)$ has an isometric copy in the James tree space $J\left(\{0,1\}^{<\omega}\right)$, thus (i) follows. And (ii) is proved by transfinite induction, as in Theorem 1.2.

For any $t=\left(t_{i}\right)_{i} \in T$, we define

$$
\begin{aligned}
A_{t}=\left\{t^{\prime}=\left(t_{i}^{\prime}\right)_{i} \in T ;\left|t^{\prime}\right|=|t|, t_{j}^{\prime} \in\left\{2 t_{j}, 2 t_{j}+1\right\}\right. \\
\\
\text { for any } j \text { such that } 0 \leq j \leq|t|\} .
\end{aligned}
$$

If $\theta \in \mathcal{T}$, then $A_{\theta}=\bigcup_{t \in \theta} A_{t}$ is a subtree of $T$, and $A_{\theta} \in \mathrm{WF}$ iff $\theta \in \mathrm{WF}$. If $\theta=\{s ; s \prec b\}$ where $b$ is a branch of $T$, then $J\left(A_{\theta}\right)$ is isometric to the James tree space $J\left(\{0,1\}^{<\omega}\right)$. Thus if $\theta \notin \mathrm{WF}$, then $J\left(A_{\theta}\right)^{\star}$ is not separable. We have shown

FACT 3.10. Let $\theta \in \mathcal{T}$.

(i) $J\left(A_{\theta}\right)$ does not contain an isomorphic copy of $\ell_{1}(\omega)$.

(ii) $J\left(A_{\theta}\right)$ is reflexive iff $\theta \in \mathrm{WF}$ iff $J\left(A_{\theta}\right)^{\star}$ is separable.

Then we can suppose that $U_{2}(T) \times J(T)$ is a subspace of $C(\Delta)$, and we identify $U_{2}(\theta)$ with $U_{2}(\theta) \times\{0\}$ and $J(T)$ with $\{0\} \times J(\theta)$ for any $\theta \in \mathcal{T}$. It is not difficult to see that the map $\varphi_{3}: \mathcal{T}^{2} \rightarrow \mathcal{S E}$ defined by $\varphi_{3}\left[\left(\theta, \theta^{\prime}\right)\right]=$ $U_{2}(\theta) \oplus J\left(A_{\theta}^{\prime}\right)$ is Borel. We have to check that $\varphi_{3}$ satisfies

$$
\varphi_{3}^{-1}\left(c^{-1}(\mathcal{J})\right)=\mathrm{WF} \times \mathrm{MF} .
$$

If $\left(\theta, \theta^{\prime}\right) \in \mathrm{WF} \times \mathrm{MF}$, then neither $U_{2}(\theta)$ is reflexive nor $J\left(A_{\theta^{\prime}}\right)$ contains $\ell_{1}(\omega)$. By a theorem due to E. Odell and H. P. Rosenthal ([L-T1], 2.e.7), $U_{2}(\theta) \oplus J\left(A_{\theta^{\prime}}\right)$ does not contain $\ell_{1}(\omega)$, since the cardinality of $\left(U_{2}(\theta) \oplus\right.$ $\left.J\left(A_{\theta^{\prime}}\right)\right)^{\star \star}$ is the same as the one of $U_{2}(\theta) \oplus J\left(A_{\theta^{\prime}}\right)$. Moreover $\left(U_{2}(\theta) \oplus\right.$ $\left.J\left(A_{\theta^{\prime}}\right)\right)^{\star}$ is not separable since $J\left(A_{\theta^{\prime}}\right)^{\star}$ is not separable. Hence $\varphi_{3}\left(\left(\theta, \theta^{\prime}\right)\right) \in$ $c^{-1}(\mathcal{J})$.

We suppose now that $\left(\theta, \theta^{\prime}\right) \notin \mathrm{WF} \times \mathrm{MF}$. If $\theta \notin \mathrm{WF}$, then $U_{2}(\theta)$ contains $\ell_{1}(\omega)$ since $U_{2}(\theta) \simeq U$, thus $\varphi_{3}\left(\left(\theta, \theta^{\prime}\right)\right) \notin c^{-1}(\mathcal{J})$. If $\theta \in \mathrm{WF}$ and $\theta^{\prime} \in \mathrm{WF}$, then $\varphi_{3}\left(\left(\theta, \theta^{\prime}\right)\right)$ is reflexive, thus $\varphi_{3}\left(\left(\theta, \theta^{\prime}\right)\right)^{\star}$ is separable, and $\varphi_{3}\left(\left(\theta, \theta^{\prime}\right)\right) \notin$ $c^{-1}(\mathcal{J})$.

Consequently, $\varphi_{3}^{-1}\left(c^{-1}(\mathcal{J})\right)=\mathrm{WF} \times \mathrm{MF}$, and Lemma 3.8 and Theorem 3.7 are proved.

4. Coanalytic ranks. Let $\mathcal{G}$ be a family of separable Banach spaces which is stable under isomorphism and coanalytic. We refer to Section 0 for 
the definition of a coanalytic rank. If $\sigma$ is a coanalytic rank on $c^{-1}(\mathcal{G})$, we also say that $\sigma$ is a coanalytic rank on $\mathcal{G}$.

First we give some general results about coanalytic ranks.

Lemma 4.1. Let $H$ be a countable set, $P$ be a Polish space, and $j$ : $P \rightarrow \mathcal{P}\left(H^{<\omega}\right)$ be such that $j(x)$ is a tree on $H$. If for any $\underline{h} \in H^{<\omega}$ the subset $\{x ; \underline{h} \in j(x)\}$ is a Borel subset of $P$, then $\{x ; j(x)$ is well founded $\}$ is coanalytic, and the map ht $\circ j$ is a coanalytic rank on this subset, where ht $\circ j(x)$ is the height of $j(x)$.

Proof. Let $n \mapsto h(n)$ be an enumeration of $H$. For $s \in \omega^{<\omega}$ we denote by $h_{s} \in H^{<\omega}$ the sequence $(h(s(i)))_{i}$, and by $j^{\prime}$ the map $P \rightarrow \mathcal{T}$ defined by $j^{\prime}(x)=\left\{s \in \omega^{<\omega} ; h_{s} \in j(x)\right\}$. By assumption, for any $s \in \omega^{<\omega}$ the subset

$$
\left\{x ; s \in j^{\prime}(x)\right\}=\left\{x ; h_{s} \in j(x)\right\}
$$

is Borel, thus $j^{\prime}$ is Borel. Therefore $j^{\prime-1}(\mathrm{WF})=\{x ; j(x)$ is well founded $\}$ is coanalytic, and ht $\circ j^{\prime}$ is a coanalytic rank on this coanalytic subset (see Proposition 0.1(iv)). For every $x \in P, \operatorname{ht}\left(j^{\prime}(x)\right)=\mathrm{ht}(j(x))$ since the map $s \mapsto h_{s}$ from $j^{\prime}(x)$ to $j(x)$ is bijective and respects inclusion (i.e. $s \prec t \Rightarrow$ $\left.h_{s} \prec h_{t}\right)$. Lemma 4.1 is proved.

Let $P$ be a Polish space. Every map $d$ from $\mathcal{F}(P)$ to $\mathcal{F}(P)$ such that $d(F) \subseteq F$ for any $F \in \mathcal{F}(P)$, and $d(F) \subseteq d\left(F^{\prime}\right)$ if $F \subseteq F^{\prime}$, is called a derivation.

If $d$ is a derivation, we associate to it an ordinal index $\sigma_{d}$ defined as follows. Let $F \in \mathcal{F}(P)$. We set $F^{(0)}=F$, and inductively define, for an ordinal $\alpha$,

$$
F^{(\alpha+1)}=d\left(F^{(\alpha)}\right)
$$

and

$$
F^{(\beta)}=\bigcap_{\alpha<\beta} F^{(\alpha)} \quad \text { if } \beta \text { is a limit ordinal. }
$$

Since $P$ is Polish, for some $\alpha<\omega_{1}$ we have $F^{(\alpha+1)}=F^{(\alpha)}$. We let $\sigma_{d}(F)=\min \left\{\alpha ; F^{(\alpha)}=\emptyset\right\}$ if such an ordinal exists, and $\omega_{1}$ otherwise.

We give without proof the following theorem. The first part is a classical result of descriptive set theory, and following the same lines as in the proof of this first part, it is not difficult to show the second part ([KL1], Chapter VI, Section 1, Theorem 4).

TheOREM 4.2. Let $K$ be a metrizable compact set. Equip $\mathcal{F}(K)$ with the Hausdorff topology.

(i) If $d$ is a Borel derivation, then $\sigma_{d}$ is a coanalytic rank on the coanalytic subset $\left\{F ; \sigma_{d}(F)<\omega_{1}\right\}$. 
(ii) Let $\left\{d_{n} ; n \in \omega\right\}$ be a countable family of Borel derivations. Then $F \mapsto \sup \left\{\sigma_{d_{n}}(F) ; n \in \omega\right\}$ defines a coanalytic rank on the coanalytic subset $\left\{F \in \mathcal{F}(K) ; \forall n \in \omega, \sigma_{d_{n}}(F)<\omega_{1}\right\}$.

Our next statement is a quantitative version of Proposition 2.8.

Proposition 4.3. The notations and assumptions are those of Proposition 2.8 .

(iii) Let $C \subseteq E$ be such that $c_{1}^{-1}(C)$ is coanalytic, and let $\sigma: C \rightarrow \omega_{1}$ be a map. Then

$$
\begin{aligned}
& \sigma \circ c_{1} \text { is a coanalytic rank on } c_{1}^{-1}(C) \text { iff } \\
& \sigma \circ c_{2} \text { is a coanalytic rank on } c_{2}^{-1}(C) .
\end{aligned}
$$

The proof is a direct application of the definition of a coanalytic rank, and of Proposition 2.8. The details are left to the reader.

We now give examples of natural coanalytic ranks on separable Banach spaces. Our first set of examples consists of the ranks of embedding. Roughly speaking, these ranks measure how long it takes to realize that a fixed space does not embed into a given Banach space.

Let $X$ be a separable Banach space with a basis, and let $\underline{x}$ be a fixed basis of $X$. We define a rank of embedding $r_{X}: \mathcal{S E} \rightarrow\left[0, \omega_{1}\right]$ such that $r_{X}(Y)=\omega_{1}$ iff $X \subset Y$. Let $Y \in \mathcal{S E}$ and $k \in \omega^{\star}$.

We denote by $T_{k}(Y)$ the tree of finite sequences $\left(z_{i}\right)_{i=0}^{n}$ in $Y$ such that $\left(z_{i}\right)_{i=0}^{n} \sim_{k}\left(x_{i}\right)_{i=0}^{n}$, and $T(Y)=\{\emptyset\} \cup \bigcup_{k \in \omega^{\star}}\left((k) \frown T_{k}(Y)\right)$ is a tree on $\omega^{\star} \cup Y$.

It is not difficult to see that $X \subset Y$ if and only if $T(Y)$ is not well founded. We will show (after stating Lemma 4.7) the following claim: if $T(Y)$ is well founded, then $\operatorname{ht}(T(Y))<\omega_{1}$. We now define the rank of embedding $r_{X}$ by $r_{X}(Y)=\operatorname{ht}(T(Y))$ if $T(Y)$ is well founded, and $\omega_{1}$ if not. Clearly $r_{X}(Y)=r_{X}(Z)$ if $Y \simeq Z$.

THEOREM 4.4. The index $r_{X}$ is a coanalytic rank on the coanalytic family $\mathcal{G}_{X}$ of all separable Banach spaces which do not contain an isomorphic copy of $X$.

In particular if $\underline{x}$ is the canonical basis of $\ell_{1}(\omega)$, we obtain an index $r_{\ell}$ which is a coanalytic rank on $\mathcal{G}_{\ell}$, and if $\underline{x}$ is a basis of $C(\Delta)$, then $r_{C(\Delta)}$ is a coanalytic rank of $\mathcal{G}_{n}$.

To prove Theorem 4.4, we use the coding $c_{b}$. Since $\mathcal{G}_{X}$ is coanalytic (Corollary 3.3), $c_{b}^{-1}\left(\mathcal{G}_{X}\right)=\left\{\underline{y} \in C(\Delta)^{\omega} ; X \not \subset \overline{\mathrm{sp}}(\underline{y})\right\}$ is coanalytic. Theorem 4.4 follows from the next lemma and Proposition 4.3.

Lemma 4.5. The map from $c_{b}^{-1}\left(\mathcal{G}_{X}\right)$ defined by $\underline{y} \mapsto r_{X}(\overline{\mathrm{sp}}(\underline{y}))$ is a coanalytic rank on $c_{b}^{-1}\left(\mathcal{G}_{X}\right)$. 
In order to be able to use Lemma 4.1, we first reduce $r_{X}(\overline{\operatorname{sp}}(y))$ to the height of a tree on the countable set $\omega^{\star} \cup \mathbb{Q}^{<\omega}$ through a classical perturbation result (see [L-T1], Proposition 1.a.9). For $y \in C(\Delta)^{\omega}$ and $k \in \omega^{\star}$, we define

$$
T_{k}(\underline{y})=\left\{\left(z_{i}\right)_{i=0}^{n} \in T_{k}(\overline{\mathrm{sp}}(\underline{y})) ; n \in \omega, z_{i} \in \operatorname{sp}_{\mathbb{Q}}(\underline{y})\right\}
$$

and the following tree on $\mathbb{Q}^{<\omega}$ :

$$
\left.T_{k}^{\prime}(\underline{y})=\left\{\underline{\mu}^{0}, \underline{\mu}^{1}, \ldots, \underline{\mu}^{n}\right) ; n \in \omega,\left(\underline{\mu}^{i} \cdot \underline{y}\right)_{i=0}^{n} \in T_{k}(\underline{y})\right\} .
$$

Then $T(\underline{y})=\{\emptyset\} \cup\left(\bigcup_{k \in \omega^{\star}}(k) \frown T_{k}(\underline{y})\right)$ is a tree on $\omega^{\star} \cup \operatorname{sp}_{\mathbb{Q}}(\underline{y})$, and $T^{\prime}(\underline{y})=$ $\{\emptyset\} \cup\left(\bigcup_{k \in \omega^{\star}}(k) \smile T_{k}^{\prime}(\underline{y})\right)$ is a tree on $\omega^{\star} \cup \mathbb{Q}^{<\omega}$.

Lemma 4.6. For any $\underline{h} \in\left(\omega^{\star} \cup \mathbb{Q}^{<\omega}\right)^{<\omega}$, the subset $\alpha(\underline{h})=\left\{y \in C(\Delta)^{\omega}\right.$; $\left.\underline{h} \in T^{\prime}(\underline{y})\right\}$ is Borel.

Indeed, if $\underline{h}=\left(k, \underline{\mu}^{0}, \underline{\mu}^{1}, \ldots, \underline{\mu}^{n}\right)$, then $\underline{y} \in \alpha(\underline{h})$ iff $\left(\underline{\mu}^{i} \cdot \underline{y}\right)_{i=0}^{n} \sim_{k}\left(x_{i}\right)_{i=0}^{n}$. Lemma 4.6 follows.

Lemma 4.7. For any $\underline{y} \in C(\Delta)^{\omega}$, we have $r_{X}(\overline{\mathrm{sp}}(\underline{y}))=\mathrm{ht}\left(T^{\prime}(\underline{y})\right)$.

We postpone for a moment the proof of this technical lemma. Note that using it, we see that if $Y$ is a separable Banach space such that $T(Y)$ is well founded, and if $y$ is a sequence such that $\overline{\mathrm{sp}}(y)=Y$, then $r_{X}(Y)=$ $\operatorname{ht}(T(Y))=\operatorname{ht}\left(T^{\prime}(y)\right)<\omega_{1}$, since $T^{\prime}(y)$ is a tree on a countable set. This proves the claim we made before stating Theorem 4.4.

Proof of Lemma 4.5. By Lemmas 4.6 and 4.1, the subset $\left\{\underline{y} \in C(\Delta)^{\omega}\right.$; $T^{\prime}(y)$ is well founded $\}$ is coanalytic, and admits as coanalytic rank the map $\underline{y} \mapsto \operatorname{ht}\left(T^{\prime}(\underline{y})\right)$, that is to say, by Lemma 4.7, the map $\underline{y} \mapsto r_{X}(\overline{\mathrm{sp}}(\underline{y}))$. Since

$$
c_{b}^{-1}\left(\mathcal{G}_{X}\right)=\left\{\underline{y} ; r_{X}(\overline{\mathrm{sp}}(\underline{y}))<\omega_{1}\right\}=\left\{\underline{y} ; T^{\prime}(\underline{y}) \text { is well founded }\right\} .
$$

Lemma 4.5 follows and Theorem 4.4 is proved.

It remains to prove Lemma 4.7.

Proof of Lemma 4.7. Let $M$ be the basis constant of $\underline{x}$. The following fact is proved in the same way as Proposition 1.a.9 of [L-T1] and we leave the proof to the reader.

FACT 4.8. Let $J$ be a subset of $\omega$, and $k \in \omega^{\star}$. If two sequences $\left(z_{n}\right)_{n \in J}$ and $\left(z_{n}^{\prime}\right)_{n \in J}$ in $C(\Delta)$ satisfy $\left(z_{n}\right)_{n \in J} \sim_{k}\left(x_{n}\right)_{n \in J}$ and for any $n \in J$,

$$
\left\|z_{n}-z_{n}^{\prime}\right\| \leq \frac{1}{2 M k} \cdot \frac{1}{2^{n+2}},
$$

then $\left(z_{n}^{\prime}\right)_{n \in J} \sim_{2 k}\left(x_{n}\right)_{n \in J}$.

Let $\underline{y} \in C(\Delta)^{\omega}$. Since $T(\underline{y}) \subseteq T(\overline{\mathrm{sp}}(y))$, if $T(\overline{\mathrm{sp}}(\underline{y}))$ is well founded, then $T(y)$ is well founded and ht $(\bar{T}(y)) \leq \operatorname{ht}(T(\overline{\operatorname{sp}}(y)))$. 
We will define a map $\ell: T(\overline{\operatorname{sp}}(y)) \rightarrow T(y)$ respecting inclusion (i.e. $s \prec t \Rightarrow \ell(s) \prec \ell(t)$ ). Thus by a classical result (see [K-L1], p. 141) if $T(y)$ is well founded, then $T(\overline{\mathrm{sp}}(y))$ is well founded and $\operatorname{ht}(T(\overline{\mathrm{sp}}(y))) \leq \operatorname{ht}(T(y))$. For every $(z, n, k) \in C(\Delta) \times \omega \times \omega^{\star}$, we pick $z(n, k) \in \operatorname{sp}_{\mathbb{Q}}(\underline{y})$ such that

$$
\|z-z(n, k)\| \leq \frac{1}{2 M k} \cdot \frac{1}{2^{n+2}} .
$$

Then we set $\ell(\emptyset)=\emptyset$; for any $k \in \omega^{\star}, \ell((k))=(2 k)$; and for any $\zeta=$ $\left(k, z_{1}, \ldots, z_{n}\right) \in T(\overline{\mathrm{sp}}(y)), \ell(\zeta)=\left(2 k, z_{1}(1, k), z_{2}(2, k), \ldots, z_{n}(n, k)\right)$. Then $\ell$ clearly respects inclusion, and $\ell(\zeta) \in T(\underline{y})$ by Fact 4.8. Consequently, $T(\underline{y})$ is well founded iff $T(\overline{\mathrm{sp}}(\underline{y}))$ is well founded, and $r_{X}(\overline{\mathrm{sp}}(\underline{y}))=\operatorname{ht}(T(\underline{y}))$. To show that ht $(T(y))=\operatorname{ht}\left(T^{\prime}(y)\right)$ for every $z \in \operatorname{sp}_{\mathbb{Q}}(y)$, we pick $\mu(z) \in$ $\mathbb{Q}^{<\omega}$ such that $\mu(z) \cdot \underline{y}=z$. Then the map from $T(\underline{y})$ to $T^{\prime}(\underline{y})$ defined by $\left.\left(k, z_{0}, z_{1}, \ldots, \bar{z}_{n}\right) \mapsto \overline{(k, \mu}\left(z_{0}\right), \underline{\mu}\left(z_{1}\right), \ldots, \underline{\mu}\left(z_{n}\right)\right)$ clearly respects inclusion. And so does the map from $T^{\prime}(\underline{y})$ to $T(\underline{y})$ defined by $\left(k, \underline{\mu}^{0}, \underline{\mu}^{1}, \ldots, \underline{\mu}^{n}\right) \mapsto$ $\left(k, \underline{\mu}^{0} \cdot \underline{y}, \underline{\mu}^{1} \cdot \underline{y}, \ldots, \underline{\mu}^{n} \cdot \underline{y}\right)$. Thus $\mathrm{ht}(Y(\underline{y}))=\operatorname{ht}\left(T^{\prime}(\underline{y})\right)$. Lemma 4.7 is proved.

When a separable Banach space $X$ is generated by a sequence $\underline{x}$ which is not a basis, we cannot use Fact 4.8 since there is no analogue of Proposition 1.a.9 of [L-T1]. However, we can still define a coanalytic rank on $\mathcal{G}_{X}$ in a similar manner. The proof is not difficult but slightly longer. We leave the details to the reader (see [Bos], Theorem 4.8) and just outline the argument.

We fix an enumeration $m \mapsto \underline{\lambda}^{m}=\left(\lambda_{i}^{m}\right)_{i}$ of $\mathbb{Q}^{<\omega}$ such that the length $\left|\underline{\lambda}^{m}\right|$ of the sequence $\underline{\lambda}^{m}$ satisfies $\left|\underline{\lambda}^{m}\right| \leq m$. We define a rank of embedding $r_{X}^{\prime}: \mathcal{S E} \rightarrow\left[0, \omega_{1}\right]$ such that $r_{X}^{\prime}(Y)=\omega_{1}$ iff $X \subset Y$ as follows. Let $Y \in \mathcal{S E}$. For any $k \in \omega^{\star}$, we denote by $A_{k}(Y)$ the tree on $Y^{<\omega}$ consisting of the empty sequence and of sequences $\left(\left(z_{0}^{0}\right),\left(z_{0}^{1}, z_{1}^{1}\right),\left(z_{0}^{2}, z_{1}^{2}, z_{2}^{2}\right), \ldots,\left(z_{0}^{n}, z_{1}^{n}, z_{2}^{n}\right)\right)$, $n \in \omega^{\star}$, such that

(1) for any $i, j, j^{\prime} \in \omega$ such that $0 \leq i \leq j \leq j^{\prime} \leq n$,

$$
\left\|z_{i}^{j}-z_{i}^{j^{\prime}}\right\| \leq \frac{k}{2 j}
$$

(2) for any $m, j \in \omega$ such that $0 \leq m \leq j \leq n$,

$$
k^{-1}\left\|\sum_{i} \lambda_{i}^{m} z_{i}^{j}\right\| \leq\left\|\sum_{i} \lambda_{i}^{m} x_{i}\right\| \leq k\left\|\sum_{i} \lambda_{i}^{m} z_{i}^{j}\right\| .
$$

Then the tree $A(Y)$ on $\omega^{\star} \cup Y^{<\omega}$ is defined by

$$
A(Y)=\{\emptyset\} \cup\left[\bigcup_{k \in \omega^{\star}}(k)^{\frown} A_{k}(Y)\right]
$$

and $r_{X}^{\prime}(Y)$ is the height of $A(Y)$ if $A(Y)$ is well founded, and $\omega_{1}$ if not.

Using this approach and the same notation, we can state, for an arbitrary separable Banach space $X$ : 
TheOREM 4.9. (i) The tree $A(Y)$ is not well founded iff $X \subset Y$.

(ii) The index $r_{X}^{\prime}$ is a coanalytic rank on $\mathcal{G}_{X}$.

The proof, which is similar to that of Theorem 4.4, is left to the reader.

Our second family of coanalytic ranks consists of the Szlenk indices, which we now define. We refer to [G-2] for a recent survey on this notion.

Let $X$ be a separable Banach space and $\varepsilon>0$. We define two derivations on $\mathcal{F}\left(B_{X^{\star}}\right)$ by

$$
\begin{aligned}
\delta(\varepsilon): F \mapsto F_{\varepsilon}^{[l]}=\left\{x^{\star} \in F ;\|\cdot\|-\operatorname{diam}(H \cap F) \geq \varepsilon\right. \\
\left.\quad \text { for all } w^{\star} \text {-open half-spaces } H \ni x^{\star}\right\}, \\
\begin{aligned}
d(\varepsilon): F \mapsto F_{\varepsilon}^{\prime}=\left\{x^{\star} \in F ;\|\cdot\|-\operatorname{diam}(V \cap F) \geq \varepsilon\right. \\
\left.\quad \text { for all } w^{\star} \text {-open sets } V \ni x^{\star}\right\},
\end{aligned}
\end{aligned}
$$

that is to say, $F_{\varepsilon}^{\prime}$ (resp. $F_{\varepsilon}^{[]]}$) is what is left from $F$ when all $w^{\star}$-open subsets (resp. $w^{\star}$-open slices) of diameter less than $\varepsilon$ are removed.

We set $\zeta_{\varepsilon}=\sigma_{d(\varepsilon)}, \xi_{\varepsilon}=\sigma_{\delta(\varepsilon)}$ and

$$
\zeta(F)=\sup _{\varepsilon>0} \zeta_{\varepsilon}(F)\left(=\sup _{\varepsilon \in \mathbb{Q}^{\star+}} \zeta_{\varepsilon}(F)\right), \quad \xi(F)=\sup _{\varepsilon>0} \xi_{\varepsilon}(F)\left(=\sup _{\varepsilon \in \mathbb{Q}^{\star+}} \xi_{\varepsilon}(F)\right) .
$$

Let now $\operatorname{Sz}(X)=\zeta\left(B_{X^{\star}}\right)$ and $\tau(X)=\xi\left(B_{X^{\star}}\right)$.

The index Sz, which is usually called the Szlenk index, has been introduced by W. Szlenk in $[\mathrm{Sz}]$. The index $\tau$ is called the dentability index. It is clear that if $X \simeq Y$, then $\mathrm{Sz}(X)=\mathrm{Sz}(Y)$ and $\tau(X)=\tau(Y)$. If $Y \subset X$, then $\mathrm{Sz}(Y) \leq \mathrm{Sz}(X)$ and $\tau(Y) \leq \tau(X)$.

Proposition 4.10. Let $X$ be a separable Banach space. The following assertions are equivalent:

(i) $X^{\star}$ is separable,

(ii) $\mathrm{Sz}(X)<\omega_{1}$,

(iii) $\tau(X)<\omega_{1}$.

This proposition is a classical application of Baire's theorem and of a result due to I. Namioka and R. Phelps ([N-P] or see [D-G-Z], Theorem I-5-2, or $[\mathrm{G}-2])$.

TheOREM 4.11. The indices $\mathrm{Sz}$ and $\tau$ are both coanalytic ranks on the family $\mathcal{G}_{s}$ of all Banach spaces with a separable dual space.

Clearly we have $\mathrm{Sz}(X) \leq \tau(X)$, and by Proposition 0.1 we obtain the following quantitative version of Proposition 4.10.

COROLlary 4.12. There exists a universal function $\psi: \omega_{1} \rightarrow \omega_{1}$ such that if $\alpha<\omega_{1}$ and if $X$ is a separable Banach space which satisfies $\operatorname{Sz}(X) \leq \alpha$, then $\tau(X) \leq \psi(\alpha)$. 
G. Lancien shows in [La] (see [La1] and [La2]) that Corollary 4.12 is true, with the same function $\psi$, for arbitrary Banach spaces, and uses it as a tool for obtaining renorming results.

Proof of Theorem 4.11. The proof is the same for $\mathrm{Sz}$ and $\tau$. We do it for Sz. We use the coding $c_{d}$ (see Section 2). It is sufficient to show that the index defined on $c_{d}^{-1}\left(\mathcal{G}_{s}\right)=\left\{\underline{w} \in \ell_{1}(\omega)^{\omega} ;\left(\ell_{1}(\omega) / \overline{\mathrm{sp}}(\underline{w})\right)^{\star}\right.$ separable $\}$ by $\underline{w} \mapsto \operatorname{Sz}\left(\ell_{1}(\omega) / \overline{\mathrm{sp}}(\underline{w})\right)$ is a coanalytic rank on $c_{d}^{-1}\left(\mathcal{G}_{s}\right)$. We denote by $K$ the closed unit ball of $\ell_{\infty}(\omega)$ and we equip it with the $w^{\star}$-topology. Thus $K$ is a metrizable compact set. The set $\mathcal{F}(K)$ is equipped with the Hausdorff topology.

Using compactness of $K$ it is not difficult to show by classical methods the following two lemmas. We leave the proofs to the reader.

Lemma 4.13. For $\varepsilon>0$, the derivation $d(\varepsilon)$ is Borel.

For $\underline{w} \in \ell_{1}(\omega)^{\omega}$, we denote by $K_{\underline{w}}$ the closed unit ball of the subspace $\underline{w}^{\perp}$ of $\ell_{\infty}(\omega)$.

Lemma 4.14. The map $k: \ell_{1}(\omega)^{\omega} \rightarrow \mathcal{F}(K)$ defined by $K(\underline{w})=K_{\underline{w}}$ is Borel.

Since $\zeta(F)=\sup _{\varepsilon \in \mathbb{Q}^{\star}+} \zeta_{\varepsilon}(F)$, Lemma 4.13 and Theorem 4.2 imply that $\zeta$ is a coanalytic rank on the coanalytic subset $\left\{F \in \mathcal{F}(K) ; \zeta(F)<\omega_{1}\right\}$. By a classical result, $w^{\perp}$ and $\left(\ell_{1}(\omega) / \overline{\mathrm{sp}}(\underline{w})\right)^{\star}$ are isometric and $w^{\star}$-isomorphic, thus we obtain easily $\mathrm{Sz}\left(\ell_{1}(\omega) / \overline{\mathrm{sp}}(\underline{w})\right)=\zeta\left(K_{\underline{w}}\right)$.

By Proposition 0.1(iv) and Lemma 4.14, the map from $\ell_{1}(\omega)^{\omega}$ to $\left[0, \omega_{1}\right]$ defined by $\underline{w} \mapsto \zeta\left(K_{\underline{w}}\right)$ is a coanalytic rank on the coanalytic subset $k^{-1}\left(\left\{F ; \zeta(F)<\omega_{1}\right\}\right)$, which is $\left\{\underline{w} \in \ell_{1}(\omega)^{\omega} ; \operatorname{Sz}\left(\ell_{1}(\omega) / \overline{\operatorname{sp}}(\underline{w})\right)<\omega_{1}\right\}=$ $c_{d}^{-1}\left(\mathcal{G}_{s}\right)$ by Proposition 4.10 . Theorem 4.11 is proved.

We give (with an outline of proofs) some results about the RadonNikodym property (RNP). For definition and general results about RNP, we refer to [D-U], and for complete proofs of the statements below, to [Bos].

We define three indices as follows. Let $X$ be a separable Banach space, $F$ be a subset of $B_{X}$, and $\varepsilon>0$. We set

$$
\begin{aligned}
& D_{1}(\varepsilon)(F)=\{x \in F ; x \in \overline{\operatorname{conv}}(F \backslash B(x, \varepsilon))\}, \\
& D_{2}(\varepsilon)(F)=\{x \in F ; \operatorname{diam}(H \cap F)>\varepsilon \text { for all open half space } H \ni x\}, \\
& D_{3}(\varepsilon)(F)=\left\{x \in F ; \forall \varepsilon^{\prime} \in(0, \varepsilon), x \in \overline{\operatorname{conv}}\left(F \backslash B\left(x, \varepsilon^{\prime}\right)\right)\right\} .
\end{aligned}
$$

In the same way as for a derivation, for any $i \in\{1,2,3\}$ we define the index $\varrho_{i}(\varepsilon)$ and we set $\varrho_{i}(F)=\sup _{\varepsilon>0} \varrho_{i}(\varepsilon)(F), \varrho_{i}(X)=\varrho_{i}\left(B_{X}\right)$. The definitions of the indices $\varrho_{1}$ and $\varrho_{2}$ are more natural, but if $F$ is closed, it is not clear that $D_{1}(\varepsilon)(F)$ is closed as well, and for $D_{2}(\varepsilon)$, if $X^{\star}$ is not separable a problem occurs with the cardinality of the set of open half spaces. The index $\varrho_{3}$ looks more convenient, and in fact we have: 
Lemma 4.15. Let $X$ be a separable Banach space. Then $\varrho_{1}(X)=\varrho_{2}(X)$ $=\varrho_{3}(X)$.

In the following, we denote this index by $\varrho(X)$. If $X \simeq Y$, we clearly have $\varrho(X)=\varrho(Y)$. It is not clear if $\varrho$ is a coanalytic rank on $\mathcal{G}_{p}$. But we have the following result which shows that $\varrho$ has some properties of coanalytic ranks.

TheOrem 4.16. (i) A separable Banach space $X$ has $R N P$ iff $\varrho(X)<\omega_{1}$.

(ii) If $\mathcal{A}$ is an analytic family of separable Banach spaces with $R N P$, which is stable under isomorphism, then there is some ordinal $\alpha<\omega_{1}$ such that $\varrho(X) \leq \alpha$ for any $X$ of the family.

Sketch of proof. (i) is a direct consequence of the definition of $\varrho$ and RNP. To prove (ii), we need the following lemma.

Lemma 4.17. For $\varepsilon>0$, the derivation $D_{3}(\varepsilon)$ from $\mathcal{F}\left(B_{C(\Delta)}\right)$ to $\mathcal{F}\left(B_{C(\Delta)}\right)$ is such that $\{(F, G) ; G \subseteq D(\varepsilon)(F)\}$ is analytic.

With this lemma, we can apply the following Theorem 4.18 on transfinite uniform boundedness, due to C. Dellacherie ([D]).

Theorem 4.18. Let $P$ be a Polish space. Equip the set $\mathcal{F}(P)$ with the Effros-Borel structure. Let $D: \mathcal{F}(P) \rightarrow \mathcal{F}(P)$ be a derivation, and $\varrho_{D}$ the associated index. If $\left\{(F, G) \in \mathcal{F}(P)^{2} ; G \subseteq D(F)\right\}$ is an analytic subset, then

(i) $C=\left\{F ; \varrho_{D}(F)<\omega_{1}\right\}$ is coanalytic.

(ii) If $A \subseteq C$ is analytic, then there is some ordinal $\alpha<\omega_{1}$ such that $\varrho_{D}(F) \leq \alpha$ for any $F \in A$.

Now let $\mathcal{A}$ be a family satisfying the assumption of Theorem 4.16(ii). Then $A=\left\{B_{X} ; X \in c^{-1}(\mathcal{A})\right\}$ is analytic since the map $\mathcal{S E} \rightarrow \mathcal{F}\left(B_{C(\Delta)}\right)$ defined by $X \mapsto B_{X}$ is Borel. For any $\varepsilon \in \mathbb{Q}^{\star+}$, by Lemma 4.17 and Theorem 4.18 there is some ordinal $\alpha_{\varepsilon}<\omega_{1}$ such that $\sup _{F \in A} \varrho(\varepsilon)(F) \leq \alpha_{\varepsilon}$. Then

$$
\sup _{F \in A} \varrho(F)=\sup _{F \in A} \sup _{\varepsilon \in \mathbb{Q}^{\star+}} \varrho(\varepsilon)(F) \leq \sup _{\varepsilon \in \mathbb{Q}^{\star+}} \alpha_{\varepsilon}=\alpha<\omega_{1}
$$

and

$$
\sup _{X \in c^{-1}(\mathcal{A})} \varrho(X)=\alpha<\omega_{1} .
$$

Theorem 4.16 follows.

In [Lo] H. P. Lotz has shown that if $X$ is a closed subspace of a Banach space with an unconditional basis, then $X^{\star}$ is separable iff $X$ does not contain $\ell_{1}(\omega)$ isomorphically, or in other words $\operatorname{Sz}(X)<\omega_{1}$ iff $r_{\ell}(X)<\omega_{1}$ where $r_{\ell}$ is the rank of embedding of $\ell_{1}(\omega)$. It is not difficult to see that 
$\{X \in \mathcal{S E} ; \exists Y \in \mathcal{S E}$ such that $X \subset Y$ and $Y$ has an unconditional basis $\}$ is analytic. Then by Proposition 0.1(iii) we obtain

THEOREM 4.19. There exist two universal functions $\psi_{1}: \omega_{1} \rightarrow \omega_{1}$ and $\psi_{2}: \omega_{1} \rightarrow \omega_{1}$ such that, if $X$ is a closed subspace of a Banach space with an unconditional basis, and if $\mathrm{Sz}(X) \leq \alpha<\omega_{1}$ and $r_{\ell}(X) \leq \beta<\omega_{1}$, then $r_{\ell}(X) \leq \psi_{1}(\alpha)$ and $\mathrm{Sz}(X) \leq \psi_{2}(\beta)$.

Remark. Without using trees on $\omega$, A. Sersouri ([Se]) has shown that there is a family of Banach spaces with separable dual on which $r_{\ell}$ is bounded by a countable ordinal, and $\mathrm{Sz}$ is not. That proves, without giving an explicit construction, that there is a Banach space with separable dual which does not contain $\ell_{1}(\omega)$ isomorphically.

We refer to [Bos3] and [B-L] for other applications of coanalytic ranks to Banach space theory.

5. Coding of basic sequences up to equivalence. We wish to develop, for basic sequences and equivalence between bases, a theory similar to what we did for separable spaces and isomorphisms. To do that, we first need a universal space. We use the basis $\underline{u}$ of Pełczyński's universal space $U$ (see Theorem 1.1) to define a coding of basic sequences up to equivalence. We determine the topological complexity of some families of basic sequences, such as "shrinking" or "boundedly complete" basic sequences. Using the Szlenk indices of the space and its dual space, we deduce a coanalytic rank on the family of reflexive Banach spaces with a basis.

We denote by $\mathcal{P}(\omega)$ the set of subsets of $\omega$. For $P \in \mathcal{P}(\omega)$ we denote by $(P(i))$ the increasing sequence of its elements, and $U_{P}$ is the closed subspace of $U$ generated by the basis $\underline{u}_{P}=\left(u_{P(i)}\right)_{i}$. By Theorem 1.1, for any basic sequence $\left(x_{i}\right)_{i}$, there is some $P \in \mathcal{P}(\omega)$ such that $\underline{u}_{P} \sim\left(x_{i}\right)_{i}$. The equivalence relation $\sim$ on $\mathcal{P}(\omega)$ is defined by $P \sim \mathcal{Q}$ iff $\underline{u}_{P} \sim \underline{u}_{\mathcal{Q}}$, and we denote by $\langle P\rangle$ the equivalence class of $P$.

Definition 5.1. A coding of basic sequences up to equivalence is a map from a set $E$ onto $\mathcal{P}(\omega) / \sim$. The canonical coding is the quotient map from $\mathcal{P}(\omega)$ onto $\mathcal{P}(\omega) / \sim$.

The equivalence relation $E_{0}$ on $2^{\omega}$ defined by $E_{0}=\left\{(s, t) \in 2^{\omega} \times 2^{\omega}\right.$; $\exists n \in \omega, \forall m \geq n, s(m)=t(m)\}$ is Borel and has no analytic section. And if $E$ is an analytic equivalence relation on a Polish space $X$, and $E_{0}$ embeds into $E$ (i.e. there is a Borel injective map $f: 2^{\omega} \rightarrow X$ such that $s E_{0} t$ iff $f(s) E f(t))$, then $E$ has no analytic section. We refer the reader to [H-K-L] for a deeper discussion of Borel equivalence relations.

TheOREM 5.2. In $\mathcal{P}(\omega)^{2}$, the equivalence relation $\sim$ is Borel and $E_{0}$ embeds into $\sim$. Thus $\sim$ has no analytic section. 
Proof. Let $P, Q \in \mathcal{P}(\omega)$. Then $P \sim Q$ iff there is $K \in \omega^{\star}$ such that for all $\underline{\lambda} \in \mathbb{Q}^{<\omega}$,

$$
\frac{1}{K}\left\|\sum_{i} \lambda_{i} u_{P(i)}\right\| \leq\left\|\sum_{i} \lambda_{i} u_{Q(i)}\right\| \leq K\left\|\sum_{i} \lambda_{i} u_{P(i)}\right\| .
$$

It is not difficult to see that $\sim$ is Borel.

To show that $E_{0}$ embeds into $\sim$, we use S. Bellenot's result ([Be]): there is a basic sequence such that two subsequences are equivalent iff only a finite number of terms are distinct.

Let $L \in \mathcal{P}(\omega)$ be such that $\underline{u}_{L}$ is equivalent to Bellenot's sequence. Define a map $2^{\omega} \rightarrow \mathcal{P}(\omega)$ by $s \mapsto P_{s}$ with $P_{s}(i)=L(2 i)$ if $s(i)=0$ and $P_{s}(i)=L(2 i+1)$ if $s(i)=1$. Since this map is continuous and injective, it is an embedding of $E_{0}$ into $\sim$. Indeed, let $(s, t) \in 2^{\omega} \times 2^{\omega}$; then $(s, t) \in E_{0}$ iff there is some $n \in \omega$ such that $s(i)=t(i)$ if $i \geq n$, thus iff $\underline{u}_{P_{s}} \sim \underline{u}_{P_{t}}$.

For a family $\mathcal{S}$ of basic sequences, we denote by $\mathcal{S}(\mathcal{P})$ the subset $\left\{P \in \mathcal{P}(\omega) ; \underline{u}_{P}\right.$ is in $\left.\mathcal{S}\right\}$. In the same way as in Section 3, we define:

Definition 5.3. A family $\mathcal{S}$ of basic sequences, stable under $\sim$, is analytic (resp. coanalytic, Borel) if $\mathcal{S}(\mathcal{P})$ is analytic (resp. coanalytic, Borel).

If such a family $\mathcal{S}$ is coanalytic, an ordinal index $\sigma$ defined on basic sequences and stable under equivalence is a coanalytic rank on $\mathcal{S}$ if $P \mapsto$ $\sigma\left(\underline{u}_{P}\right)$ is a coanalytic rank on $\mathcal{S}(\mathcal{P})$.

We recall some definitions and properties relating to basic sequences which are shrinking or boundedly complete (see [L-T1]). A basis $\left(x_{i}\right)_{i \in \omega}$ of a Banach space $X$ is shrinking if the sequence $\left(x_{i^{\star}}\right)_{i \in \omega}$ of biorthogonal functionals is a basis of $X^{\star}$, and boundedly complete if for every sequence of scalars $\left(a_{i}\right)_{i \in \omega}$ such that $\sup _{n}\left\|\sum_{i=1}^{n} a_{i} x_{i}\right\|<\infty$, the series $\sum_{n=1}^{\infty} a_{n} x_{n}$ converges. The basis $\left(x_{i}\right)_{i \in \omega}$ is boundedly complete iff $X=\overline{\operatorname{sp}}\left(\left\{x_{i}^{\star} ; i \in \omega\right\}\right)^{\star}$; then we define $X_{\star}=\overline{\mathrm{sp}}\left(\left\{x_{i}^{\star} ; i \in \omega\right\}\right)$. A basic sequence $\left(x_{i}\right)_{i \in \omega}$ is shrinking or boundedly complete if it has this property in $\overline{\mathrm{sp}}\left(\left\{x_{i} ; i \in \omega\right\}\right)$.

R. C. James has shown ([J-2] or [L-T1]) that if $X$ is a Banach space with a basis $\left(x_{i}\right)_{i \in \omega}$, then $X$ is reflexive iff $\left(x_{i}\right)_{i \in \omega}$ is both shrinking and boundedly complete.

The main result of this section is the following.

THEOREM 5.4. (i) The family $\mathcal{S}_{s}$ of shrinking basic sequences is coanalytic non-Borel, and the ordinal index $\underline{x} \mapsto \mathrm{Sz}(\overline{\mathrm{sp}}(\underline{x}))$ is a coanalytic rank on $\mathcal{S}_{s}$.

(ii) The family $\mathcal{S}_{b}$ of boundedly complete basic sequences is coanalytic non-Borel, and the ordinal index $\underline{x} \mapsto \mathrm{Sz}\left((\overline{\mathrm{sp}}(\underline{x}))_{\star}\right)$ is a coanalytic rank on $\mathcal{S}_{b}$. In fact, $\mathcal{S}_{s}(\mathcal{P})$ and $\mathcal{S}_{b}(\mathcal{P})$ are complete coanalytic subsets. 
COROLlary 5.5. The family $\mathcal{S}_{r}$ of bases of reflexive spaces is coanalytic non-Borel, and the ordinal index $\underline{x} \mapsto \sup \left(\operatorname{Sz}(\overline{\operatorname{sp}}(\underline{x})), \operatorname{Sz}\left(\overline{\operatorname{sp}}(\underline{x})^{\star}\right)\right)$ defines a coanalytic rank on $\mathcal{S}_{r}$. In fact $\mathcal{S}_{r}(\mathcal{P})$ is a complete coanalytic subset.

We do not know if the ordinal index defined by $\sup \left(\mathrm{Sz}(X), \operatorname{Sz}\left(X^{\star}\right)\right)$ when $X$ is a separable reflexive space is a coanalytic rank on the family $\mathcal{G}_{r}$ of separable reflexive Banach spaces.

The Szlenk index of a separable reflexive Banach space does not control the Szlenk index of its dual space. In [La] G. Lancien has shown that the family of reflexive spaces $\left\{X_{\alpha} ; \alpha<\omega_{1}\right\}$ considered by W. Szlenk ([Sz]) satisfies, for every $\alpha<\omega_{1}, \operatorname{Sz}\left(X_{\alpha}\right) \geq \alpha$ and $\operatorname{Sz}\left(X_{\alpha}^{\star}\right) \leq \omega$. It follows that a separable Banach space which contains an isomorphic copy of every reflexive space with a Szlenk index less than $\omega$ does not have RNP.

We now proceed to the proof of Theorem 5.4. This is done through several lemmas.

Lemma 5.6. The subsets $\mathcal{S}_{s}(\mathcal{P}), \mathcal{S}_{b}(\mathcal{P})$ and $\mathcal{S}_{r}(\mathcal{P})$ of $\mathcal{P}(\omega)$ are not Borel.

Proof. We use the family $\left\{U_{2}(\theta) ; \theta \in \mathcal{T}\right\}$ (see Section 1 ). Let $P_{T} \in \mathcal{P}(\omega)$ be such that $\underline{u}_{P_{T}}$ is equivalent to the basis $\left\{\chi_{s_{i}} ; i \in \omega\right\}$ of $U_{2}(T)$. For $\theta \in \mathcal{T}$ we set $P_{\theta}=\left\{P_{T}(i) ; s_{i} \in \theta\right\}$. Thus $\underline{u}_{P_{\theta}} \sim\left(\chi_{s_{i}} ; s_{i} \in \theta\right)$ and $U_{P_{\theta}} \simeq U_{2}(\theta)$.

The map $\varphi_{b}: \mathcal{T} \rightarrow \mathcal{P}(\omega)$ defined by $\varphi_{b}(\theta)=P_{\theta}$ is clearly continuous, and

$$
\varphi_{b}^{-1}\left(\mathcal{S}_{s}(\mathcal{P})\right)=\varphi_{b^{-1}}\left(\mathcal{S}_{b}(\mathcal{P})\right)=\varphi_{b}^{-1}\left(\mathcal{S}_{r}(\mathcal{P})\right)=\mathrm{WF} .
$$

Indeed, if $\theta \in \mathrm{WF}$, then $U_{P_{\theta}} \simeq U_{2}(\theta)$ is reflexive, thus $\underline{u}_{P_{\theta}}$ is shrinking and boundedly complete, and $P_{\theta} \in \mathcal{S}_{s}(\mathcal{P}) \cap \mathcal{S}_{b}(\mathcal{P}) \cap \mathcal{S}_{r}(\mathcal{P})$. If $\theta \notin \mathrm{WF}$, then $U_{P_{\theta}} \simeq U_{2}(\theta) \simeq U$, thus $\underline{u}_{P_{\theta}}$ is neither shrinking nor boundedly complete, and $P_{\theta}$ is neither in $\mathcal{S}_{s}(\mathcal{P})$ nor in $\mathcal{S}_{b}(\mathcal{P})$ nor in $\mathcal{S}_{r}(\mathcal{P})$. Lemma 5.6 follows since $\mathrm{WF}$ is not Borel.

We can show that $\mathcal{S}_{s}, \mathcal{S}_{b}$ and $\mathcal{S}_{r}$ are coanalytic by classical methods, but it will follow from results about coanalytic ranks.

We need a more general lemma about the convergence index (see [K-L2]), which is defined as follows. Let $X$ be a Banach space, $K$ be a compact metric space and $\left(f_{m}\right)_{m \in \omega}$ be a sequence of continuous functions from $K$ to $X$. The set $\mathcal{F}(K)$ of closed subsets of $K$ is equipped with the Hausdorff topology. For any $\varepsilon>0, D_{c}(\varepsilon)$ is the derivation on $\mathcal{F}(K)$ defined by

$$
\begin{aligned}
D_{c}(\varepsilon)(F)= & \left\{x \in F ; \forall V \ni x \text { open subset, } \forall N \in \omega^{\star},\right. \\
& \left.\exists m \geq N, n \geq N, x^{\prime} \in V \cap F \text { such that }\left\|f_{m}\left(x^{\prime}\right)-f_{n}\left(x^{\prime}\right)\right\| \geq \varepsilon\right\} .
\end{aligned}
$$

We denote by $\gamma_{\varepsilon}$ the associated index (see Section 4 ), and the convergence index $\gamma$ of $\left(f_{m}\right)_{m \in \omega}$ is

$$
\gamma(F)=\sup _{\varepsilon>0} \gamma_{\varepsilon}(F)=\sup _{\varepsilon \in \mathbb{Q}^{\star+}} \gamma_{\varepsilon}(F)
$$


It is classical that $\gamma(F)<\omega_{1}$ iff $\left(f_{m}\right)_{m \in \omega}$ pointwise converges on $F$.

Lemma 5.7. (i) For $\varepsilon>0$, the derivation $D_{c}(\varepsilon)$ is Borel.

(ii) The convergence index $\gamma$ is a coanalytic rank on the coanalytic subset $\left\{F \in \mathcal{F}(K) ;\left(f_{m}\right)_{m \in \omega}\right.$ pointwise converges on $\left.F\right\}$.

Proof. The proof of (i) is done by classical methods and we leave it to the reader. Then (ii) follows by Theorem 4.2 .

For $n \in \omega$ we denote by $\Pi_{n}$ the projection from $U$ onto $\operatorname{sp}\left(\left\{u_{i} ; i \leq n\right\}\right)$ defined by $\Pi_{n}\left(u_{i}\right)=u_{i}$ if $i \leq n$, and 0 if not. Then $\Pi_{n}^{\star}: U^{\star} \rightarrow U^{\star}$ satisfies $\Pi_{n}^{\star}\left(u_{i}^{\star}\right)=u_{i}^{\star}$ if $i \leq n$, and 0 if not. And $\Pi_{n}^{\star}$ is $w^{\star}$-continuous since $\Pi_{n}$ is continuous. Since the range of $\Pi_{n}^{\star}$ is finite-dimensional, $\Pi_{n}^{\star}$ is continuous from $\left(U^{\star}, w^{\star}\right)$ to $\left(U^{\star},\|\cdot\|\right)$. We denote by $K_{U^{\star}}$ the compact metric set $\left(B_{U^{\star}}, w^{\star}\right)$. Then $\Pi_{n}^{\star}: K_{U^{\star}} \rightarrow\left(U^{\star},\|\cdot\|\right)$ is continuous.

In the following, we denote by $\gamma$ the convergence index of $\left(\Pi_{n}^{\star}\right)_{n \in \omega}$. By Lemma 5.7 we obtain

FACT 5.8. The subset of $\mathcal{F}\left(K_{U^{\star}}\right)$ defined by $\left\{F ; \gamma(F)<\omega_{1}\right\}=$ $\left\{F ;\left(\Pi_{n}^{\star}\right)_{n \in \omega}\right.$ pointwise converges on $\left.F\right\}$ is coanalytic, and $\gamma$ is a coanalytic rank on this subset.

We will use a coding which is slightly different from the canonical coding, but equivalent by Propositions 2.8 and 4.3. Let $\mathcal{P}_{0}=\{P \in \mathcal{P}(\omega)$; $\underline{u}_{P}$ is complemented $\}$. By Theorem 1.1, for every basic sequence $\underline{x}$, there is some $P \in \mathcal{P}_{0}$ such that $\underline{u}_{P} \sim \underline{x}$. Thus the map $\mathcal{P}_{0} \rightarrow \mathcal{P}(\omega) / \sim$ defined by $P \mapsto\langle P\rangle$ is a coding of basic sequences up to equivalence. It is not difficult to prove that $\mathcal{P}_{0}$ is Borel, and that so is $\left\{(P, Q) \in \mathcal{P}(\omega) \times \mathcal{P}_{0} ;\langle P\rangle=\langle Q\rangle\right\}$. Thus $\mathcal{P}_{0}$ is a standard Borel space, and the assumptions of Propositions 2.8 and 4.3 are fulfilled. That ensures that the coding is equivalent to the canonical coding in the sense of these propositions. We denote by $\mathcal{S}_{s}\left(\mathcal{P}_{0}\right)$, $\mathcal{S}_{b}\left(\mathcal{P}_{0}\right), \mathcal{S}_{r}\left(\mathcal{P}_{0}\right)$ the subsets $\mathcal{S}_{s}(\mathcal{P}) \cap \mathcal{P}_{0}, \mathcal{S}_{b}(\mathcal{P}) \cap \mathcal{P}_{0}$, and $\mathcal{S}_{r}(\mathcal{P}) \cap \mathcal{P}_{0}$.

Let $P \in \mathcal{P}_{0}$ and denote by $K_{P}$ the unit ball of $\overline{\mathrm{sp}}^{\star}\left(\left\{u_{i}^{\star} ; i \in P\right\}\right)$. We have $U=U_{P} \oplus U_{\omega \backslash P}$ and

$$
\overline{\mathrm{sp}}^{\star}\left(\left\{u_{i}^{\star} ; i \in P\right\}\right)=U_{\omega \backslash P}^{\perp} \simeq\left(U / U_{\omega \backslash P}\right)^{\star} \simeq U_{P}^{\star} .
$$

The spaces $U_{P}^{\star}$ and $U_{\omega \backslash P}^{\perp}$ are $\|\cdot\|$-isomorphic and $w^{\star}$-isomorphic, via an isomorphism such that the image of the sequence of biorthogonal functionals of $\underline{u}_{P}$ is the sequence $\left(u_{P(i)}^{\star}\right)_{i \in \omega}$. That justifies the coding from $\mathcal{P}_{0}$. We identify $U_{P}^{\star}$ and $\overline{\mathrm{sp}}^{\star}\left(\left\{u_{i}^{\star} ; i \in P\right\}\right)$.

Lemma 5.9. (i) The map $\varphi_{4}: \mathcal{P}_{0} \rightarrow \mathcal{F}\left(K_{U^{\star}}\right)$ defined by $\varphi_{4}(P)=K_{P}$ is Borel.

(ii) Let $P \in \mathcal{P}_{0}$. Then $\underline{u}_{P}$ is shrinking iff $\gamma\left(K_{P}\right)<\omega_{1}$.

(iii) Let $P \in \mathcal{P}_{0}$ be such that $\underline{u}_{P}$ is shrinking. Then $\gamma\left(K_{P}\right)=\operatorname{Sz}\left(U_{P}\right)$. 
Proof. (i) It is sufficient to prove that $\left\{P \in \mathcal{P}_{O} ; K_{P} \subseteq O\right\}$ is Borel when $O$ is a $w^{\star}$-open subset of $B_{U^{\star}}$. Thanks to the $w^{\star}$-compactness of $B_{U^{\star}}$, the proof is not difficult and we leave it to the reader.

(ii) The sequence $\underline{u}_{P}$ is shrinking iff

$$
\overline{\mathrm{sp}}^{\star}\left(\left\{u_{i}^{\star} ; i \in P\right\}\right)=\overline{\mathrm{sp}}\left(\left\{u_{i}^{\star} ; i \in P\right\}\right)
$$

$$
\begin{aligned}
& \text { iff }\left(\Pi_{n}^{\star}\right)_{n \in \omega} \text { pointwise converges on } K_{P} \\
& \text { iff } \gamma\left(K_{P}\right)<\omega_{1} .
\end{aligned}
$$

(iii) We refer to Section 4 for notations. Using the $w^{\star}$ and norm isomorphism between $U_{P}^{\star}$ and $\overline{\mathrm{sp}}^{\star}\left(\left\{u_{i}^{\star} ; i \in P\right\}\right)$, we obtain $\mathrm{Sz}\left(U_{P}\right)=\zeta\left(K_{P}\right)$. It is sufficient to prove that $\zeta\left(K_{P}\right)=\gamma\left(K_{P}\right)$ if $P \in \mathcal{S}_{s}\left(\mathcal{P}_{0}\right)$, i.e. if $\underline{u}_{P}$ is shrinking.

FACT 5.10. Let $P \in \mathcal{S}_{s}\left(\mathcal{P}_{0}\right), \varepsilon>0$ and $F \in \mathcal{F}\left(K_{P}\right)$. Then $F_{8 \varepsilon}^{\prime} \subseteq D_{c}(\varepsilon)$. Thus $\zeta\left(K_{P}\right) \leq \gamma\left(K_{P}\right)$.

Indeed, let $x \in F_{8 \varepsilon}^{\prime}, N \in \omega^{\star}$ and let $V \in \mathcal{V}^{\star}(x)$, the set of $w^{\star}$-neighbourhoods of $x$. Since $\underline{u}_{P}$ is shrinking, there is $n \geq N$ such that $\left\|\Pi_{n}^{\star}(x)-x\right\|$ $\leq \varepsilon$. Since $\Pi_{n}^{\star}$ is $w^{\star}-\|\cdot\|$ continuous, there exists $V_{1} \in \mathcal{V}^{\star}(x)$ such that $\left\|\Pi_{n}^{\star}\left(x^{\prime}\right)-\Pi_{n}^{\star}(x)\right\| \leq \varepsilon$ for any $x^{\prime} \in V_{1}$. Since $\operatorname{diam}\left(V \cap V_{1} \cap F\right)>8 \varepsilon$, there is $x^{\prime} \in V \cap V_{1} \cap F$ such that $\left\|x^{\prime}-x\right\| \geq 4 \varepsilon$. And there is $m \geq n$ such that $\left\|\Pi_{m}^{\star}\left(x^{\prime}\right)-x^{\prime}\right\| \leq \varepsilon$.

We easily obtain

$$
4 \varepsilon \leq\left\|x^{\prime}-x\right\| \leq 3 \varepsilon+\left\|\Pi_{m}^{\star}\left(x^{\prime}\right)-\Pi_{n}^{\star}\left(x^{\prime}\right)\right\|
$$

thus

$$
\left\|\Pi_{m}^{\star}\left(x^{\prime}\right)-\Pi_{n}^{\star}\left(x^{\prime}\right)\right\| \geq \varepsilon .
$$

Therefore, $x \in D_{c}(\varepsilon)(F)$ since

$$
\forall V \in \mathcal{V}^{\star}(x), \forall N \in \omega^{\star}, \exists m \geq N, \exists n \geq N, \exists x^{\prime} \in V \cap F
$$

such that

$$
\left\|\Pi_{m}^{\star}\left(x^{\prime}\right)-\Pi_{n}^{\star}\left(x^{\prime}\right)\right\| \geq \varepsilon .
$$

By transfinite induction, it follows that $\zeta_{8 \varepsilon}(F) \leq \gamma_{\varepsilon}(F)$, thus $\zeta(F) \leq \gamma(F)$, and $\mathrm{Sz}\left(U_{P}\right)=\zeta\left(K_{P}\right) \leq \gamma\left(K_{P}\right)$.

FACT 5.11. Let $P \in \mathcal{S}_{s}\left(\mathcal{P}_{0}\right), \varepsilon>0$ and $F \in \mathcal{F}\left(K_{P}\right)$. Then $D_{c}\left(3 c_{u} \varepsilon\right)(F)$ $\subseteq F_{\varepsilon}^{\prime}$, thus $\gamma\left(K_{P}\right) \leq \zeta\left(K_{P}\right)$, where $c_{u}$ is the basic constant of $\underline{u}$.

Indeed, let $x \in F \backslash F_{\varepsilon}^{\prime}$. There is $V \in \mathcal{V}^{\star}(x) \operatorname{such}$ that $\operatorname{diam}(V \cap F) \leq \varepsilon$, and since $\underline{u}_{P}$ is shrinking there is $N \in \omega$ such $\left\|\Pi_{m}^{\star}(x)-\Pi_{n}^{\star}(x)\right\| \leq c_{u} \varepsilon$ for any $m, n \geq N$. Since $\left\|\Pi_{n}\right\|=\left\|\Pi_{n}^{\star}\right\| \leq c_{u}$ for any $n \in \omega$, and since $\left\|x^{\prime}-x\right\| \leq \varepsilon$ for any $x^{\prime} \in V \cap F$, we obtain

$$
\begin{gathered}
\left\|\Pi_{m}^{\star}\left(x^{\prime}\right)-\Pi_{n}^{\star}\left(x^{\prime}\right)\right\| \leq \\
+\left\|\Pi_{m}^{\star}\left(x^{\prime}\right)-\Pi_{m}^{\star}(x)\right\|+\left\|\Pi_{m}^{\star}(x)-\Pi_{n}^{\star}(x)\right\| \\
+\left\|\Pi_{n}^{\star}(x)-\Pi_{n}^{\star}\left(x^{\prime}\right)\right\| \leq 3 c_{u} \varepsilon .
\end{gathered}
$$


Thus $x \notin D_{c}\left(3 c_{u} \varepsilon\right)(F)$, and $D_{c}\left(3 c_{u} \varepsilon\right)(F) \subseteq F_{\varepsilon}^{\prime}$. By transfinite induction we obtain $\gamma_{3 c_{u} \varepsilon}(F) \leq \zeta_{\varepsilon}(F)$, thus $\gamma(F) \leq \zeta(F)$ and

$$
\gamma\left(K_{P}\right) \leq \zeta\left(K_{P}\right)=\mathrm{Sz}\left(U_{P}\right) .
$$

Lemma 5.9(iii) follows.

Finally we are ready to show Theorem 5.4 and Corollary 5.5.

Proof of Theorem 5.4. (i) By Lemma 5.9 we have

$$
\begin{aligned}
\varphi_{4}^{-1}\left(\left\{F ; \gamma(F)<\omega_{1}\right\}\right. & =\left\{P \in \mathcal{P}_{0} ; \gamma\left(K_{P}\right)<\omega_{1}\right\} \\
& =\left\{P \in \mathcal{P}_{0} ; \underline{u}_{P} \text { shrinking }\right\} .
\end{aligned}
$$

By Fact 5.8 this subset is coanalytic, and $P \mapsto \gamma\left(K_{P}\right)$ is a coanalytic rank on it. Thus by Lemma 5.9 (iii), $P \mapsto \mathrm{Sz}\left(U_{P}\right)$ is a coanalytic rank on the coanalytic subset $\mathcal{S}_{s}\left(\mathcal{P}_{0}\right)$.

Since the coding from $\mathcal{P}_{0}$ is equivalent to the canonical coding in the sense of Propositions 2.8 and 4.3, it follows that $P \mapsto \mathrm{Sz}\left(U_{P}\right)$ is a coanalytic rank on the coanalytic non-Borel (by Lemma 5.6) subset $\mathcal{S}_{s}(\mathcal{P})$, and (i) follows.

(ii) Let $Q \subseteq \omega$ be such that $\underline{u}_{Q} \sim\left(u_{i}^{\star}\right)_{i \in \omega}$. For $P \in \mathcal{P}_{0}, \bar{u}_{P}$ is boundedly complete iff $\left(u_{P(i)}^{\star}\right)_{i \in \omega}$ is shrinking, by the $\|\cdot\|$ and $w^{\star}$-isomorphism between $U_{P}^{\star}$ and $\overline{\operatorname{sp}}^{\star}\left(\left\{u_{i}^{\star} ; i \in P\right\}\right)$. Since $\left.\left(u_{P(i)}^{\star}\right)_{i \in \omega} \sim\left(u_{Q(P(i))}\right)_{i \in \omega}\right), \underline{u}_{P}$ is boundedly complete iff $\left.\left(u_{Q(P(i))}\right)_{i \in \omega}\right)$ is shrinking.

It is not difficult to see that the map $\varphi_{5 i}: \mathcal{P}_{0} \rightarrow \mathcal{P}(\omega)$ defined by $\varphi_{5}(P)=Q(P)=\{Q(P(i)) ; i \in \omega\}$ is Borel.

Since $\varphi_{5}^{-1}\left(\mathcal{S}_{s}(\mathcal{P})\right)=\mathcal{S}_{b}\left(\mathcal{P}_{0}\right)$, we see that $\mathcal{S}_{b}\left(\mathcal{P}_{0}\right)$ is coanalytic, and $P \mapsto \mathrm{Sz}\left(U_{Q(P)}\right)$ is a coanalytic rank on $\mathcal{S}_{b}\left(\mathcal{P}_{0}\right)$.

When $\underline{u}_{P}$ is boundedly complete, with $P \in \mathcal{P}_{0}$, we have $U_{Q(P)} \simeq$ $\overline{\mathrm{sp}}\left(\left\{u_{i}^{\star} ; i \in P\right\}\right) \simeq\left(U_{P}\right)_{\star}$. Thus $P \mapsto \mathrm{Sz}\left[\left(U_{P}\right)_{\star}\right]$ is a coanalytic rank on $\mathcal{S}_{b}\left(\mathcal{P}_{0}\right)$. By equivalence between codings, (ii) follows.

Finally, the proof of Lemma 5.6 shows that $\mathcal{S}_{s}(\mathcal{P})$ and $\mathcal{S}_{b}(\mathcal{P})$ are complete coanalytic subsets.

Proof of Corollary 5.5. If $P \in \mathcal{P}(\omega)$ is such that $U_{P}$ is reflexive then $\left(U_{P}\right)_{\star}=U_{P}^{\star}$. Since $\mathcal{S}_{r}(\mathcal{P})=\mathcal{S}_{s}(\mathcal{P}) \cap \mathcal{S}_{b}(\mathcal{P})$, Corollary 5.5 follows. By the proof of Lemma 5.6, $\mathcal{S}_{r}(\mathcal{P})$ is a complete coanalytic subset.

6. The embedding of $E_{0}$ into the isomorphism relation. The equivalence relation $E_{0}$ (whose definition is recalled in Section 5) has no measurable selection, and the main result of $[\mathrm{H}-\mathrm{K}-\mathrm{L}]$ asserts that this relation embeds into every non-smooth Borel equivalence relation $R$ on a Polish space $P$. We recall that this means there is a Borel 1-to- 1 map $f$ from $2^{\omega}$ into $P$ such that $x E_{0} y$ if and only if $f(x) R f(y)$. As shown in Section 2, the isomorphism equivalence relation between separable Banach spaces is 
not Borel, hence we cannot apply [H-K-L]. However the following proposition shows that the result still holds. Note that this embedding provides an alternative way to show that the isomorphism relation has no analytic section.

Proposition 6.1. The equivalence relation $E_{0}$ on $2^{\omega}$ embeds into the isomorphism relation on $\mathcal{S E}$, and thus the latter has no analytic section.

To prove this result we will use the reflexive Banach space $X$ with an unconditional basic sequence and which fails the hyperplane property, built by W. T. Gowers ([Gow]). A vector $x \in X$ is denoted by $\sum_{i \in \omega} x(i) e_{i}$, and $\operatorname{supp}(x)$ is the set $\{i \in \omega ; x(i) \neq 0\}$. Let $x, y \in X$, and $n \in \omega$. The notations $x<y, x<n$ and $x>n$ mean respectively $\max (\operatorname{supp}(x))<\min (\operatorname{supp}(y))$, $\max (\operatorname{supp}(x))<n$, and $\min (\operatorname{supp}(x))>n$. The space $X$ satisfies a criterion due to P. Casazza, that is: if $\left(y_{n}\right)_{n \in \omega}$ and $\left(z_{n}\right)_{n \in \omega}$ are two sequences in $X$ such that $y_{n}<z_{n}<y_{n+1}$ for every $n \in \omega$, then they are not equivalent. The purpose of this criterion is to conclude that there is no proper subspace $Y$ of $X$ such that $Y \simeq X$. In fact we show below a slightly more general result. If $F \in \mathcal{P}(\omega)$ and $n \in \omega$, we define

$$
F(n)=\{i \in F ; i \leq n\} \quad \text { and } \quad X_{F}=\overline{\operatorname{sp}}\left\{e_{n} ; n \in F\right\} .
$$

Lemma 6.2. Let $F$ and $G$ be two infinite subsets of $\omega$ such that there is some strictly increasing sequence $\left(n_{i}\right)_{i \in \omega}$ which satisfies $\operatorname{card}\left(F\left(n_{i}\right)\right)>$ $\operatorname{card}\left(G\left(n_{i}\right)\right)$ for every $i \in \omega$. Then no subspace of $X_{G}$ is isomorphic to $X_{F}$.

Proof. Assume there is an isomorphism $T: X_{F} \rightarrow Y$ where $Y$ is a subspace of $X_{G}$. We can suppose that $\operatorname{card}\left(\operatorname{supp}\left(T e_{n}\right)\right)$ is finite for any $n \in \omega$.

Since $\operatorname{card}\left(F\left(n_{0}\right)\right)>\operatorname{card}\left(G\left(n_{0}\right)\right)$, it follows that $\operatorname{dim} T X_{F\left(n_{0}\right)}>$ $\operatorname{dim} X_{G\left(n_{0}\right)}$, thus there is $x_{0} \in F\left(n_{0}\right)$ with $\left\|x_{0}\right\|=1$ such that $T x_{0}>n_{0}$, so $x_{0}<T x_{0}$. Then there is $m_{1} \in\left\{n_{i} ; i \in \omega\right\}$ such that $m_{1}>n_{0}$ and $T x_{0}<m_{1}$. As before there is $x_{1} \in X_{F\left(m_{1}\right)}$ with $\left\|x_{1}\right\|=1$ such that $T x_{1}>m_{1}$, since $\operatorname{card}\left(F\left(m_{1}\right)\right)>\operatorname{card}\left(G\left(m_{1}\right)\right)$. Thus we have $x_{1}<T x_{1}$ and $T x_{0}<T x_{1}$. By induction we obtain a sequence $\left(x_{i}\right)_{i \in \omega}$ in $X_{F}$ such that $\left\|x_{i}\right\|=1$ and $x_{i}<T x_{i}$ for any $i \in \omega$, and $T x_{0}<T x_{1}<T x_{2}<\ldots$ The sequence $\left(T x_{i}\right)_{i \in \omega}$ is basic, and $\left(x_{i}\right)_{i \in \omega}$ is basic as well since $T$ is an isomorphism. Since $X$ is reflexive, we can apply a classical result of Bessaga and Pełczyński ([L-T1], Prop. 1.a.12) to get a subsequence $\left(x_{n_{k}}\right)_{k \in \omega}$ which is equivalent to a block-basis $\left(x_{n_{k}}^{\prime}\right)_{k \in \omega}$ of the original basis, such that $x_{n_{k}}^{\prime}<T x_{n_{k}}$. Taking a subsequence, we obtain two equivalent basic sequences $\left(y_{m}\right)_{m \in \omega}$ and $\left(z_{m}\right)_{m \in \omega}$ such that $y_{m}<z_{m}<y_{m+1}$. Thus $X$ does not satisfy the Casazza criterion, a contradiction. 
Proof of Proposition 6.1. We can suppose $X \in \mathcal{S E}$. Let $s=(s(i))_{i \in \omega}$ $\in 2^{\omega}$. We set

$$
X_{s}=\overline{\operatorname{sp}}\left\{e_{2 i+s(i)} ; i \in \omega\right\} \quad \text { and } \quad F_{s}=\{n ; n=2 i+s(i), i \in \omega\} .
$$

It is easy to verify that the map $2^{\omega} \rightarrow \mathcal{S E}$ defined by $s \mapsto X_{s}$ is Borel. Let $s, t \in 2^{\omega}$.

If $s E_{0} t$, then $X_{s}$ and $X_{t}$ have the same finite codimension in $\overline{\operatorname{sp}}\left\{X_{s} \cup X_{t}\right\}$, thus $X_{s} \simeq X_{t}$.

If $s E_{0} t$ fails, then there is a strictly increasing sequence $\left(n_{i}\right)_{i \in \omega}$ such that either $\operatorname{card}\left(F_{s}\left(n_{i}\right)\right)>\operatorname{card}\left(F_{t}\left(n_{i}\right)\right)$ for any $i \in \omega$, or $\operatorname{card}\left(F_{t}\left(n_{i}\right)\right)>$ $\operatorname{card}\left(F_{s}\left(n_{i}\right)\right)$ for any $i \in \omega$. Thus $X_{s} \not X_{t}$ by the Lemma.

Therefore $E_{0}$ embeds into $\simeq$, and it follows (see $\left.[\mathrm{H}-\mathrm{K}-\mathrm{L}]\right)$ that $\simeq$ has no analytic section in $\mathcal{S E}$.

Note that the proof of Proposition 6.1 gives an improvement of Theorem 5.2, since when $s E_{0} t$ fails, the corresponding bases are not equivalent and in fact the spaces $X_{s}$ and $X_{t}$ are not even isomorphic.

\section{References}

[Be] S. F. Bellenot, The Banach spaces of Maurey and Rosenthal and totally incomparable bases, J. Funct. Anal. 95 (1991), 96-105.

[Bos] B. Bossard, Théorie descriptive des ensembles en géométrie des espaces de Banach, thèse, Univ. Paris VI, 1994.

[Bos1] - Codages des espaces de Banach séparables. Familles analytiques ou coanalytiques d'espaces de Banach, C. R. Acad. Sci. Paris Sér. I 316 (1993), 1005-1010.

[Bos2] - An ordinal version of some applications of the classical interpolation theorem, Fund. Math. 152 (1997), 55-74.

[Bos3] -, On a problem of H. P. Rosenthal, Houston J. Math. 26 (2000), 1-16.

[B-G-K] B. Bossard, G. Godefroy and R. Kaufman, Hurewicz's theorems and renormings of Banach spaces, J. Funct. Anal. 140 (1996), 142-150.

[B-L] B. Bossard and G. Lopez, The point of continuity property: descriptive complexity and ordinal index, Serdica Math. J. 24 (1998), 199-214.

[Bou1] J. Bourgain, The Szlenk index and operators on $C(K)$ spaces, Bull. Soc. Math. Belgique 31 (1979), 87-117.

[Bou2] -, On convergent sequences of continuous functions, ibid. 32 (1980), 235-249.

[Bou3] - On separable Banach spaces, universal for all separable reflexive spaces, Proc. Amer. Math. Soc. 79 (1980), 241-246.

[Bou4] - New Classes of $\mathcal{L}^{p}$-Spaces, Lecture Notes in Math. 889, Springer, 1981.

[C] J. P. R. Christensen, Topology and Borel Structure, North-Holland Math. Stud. 10, North-Holland, 1974.

[D-G-S] G. Debs, G. Godefroy and J. Saint-Raymond, Topological properties of the set of norm-attaining linear functionals, Canad. J. Math. 47 (1993), 318-329.

[D] C. Dellacherie, Les dérivations en théorie descriptive des ensembles et le théorème de la borne, in: Séminaire de Probabilités XI, Lecture Notes in Math. 581, Springer, 1977, 34-46. 
[D-G-Z] R. Deville, G. Godefroy and V. Zizler, Smoothness and Renormings in Banach Spaces, Pitman Monographs Surveys Pure Appl. Math. 64, Longman, 1995, 318-329.

[D-U] J. Diestel and J. J. Uhl, Vector Measures, Math. Surveys 15, Amer. Math. Soc., 1977.

[G1] G. Godefroy, On analytic families of sets in harmonic analysis, Illinois J. Math. 35 (1991), 241-249.

[G2] - The Szlenk index and its applications, Nova Math. Publ., to appear.

[Gow] W. T. Gowers, A solution to Banach's hyperplane problem, Bull. London Math. Soc. 26 (1994), 523-530.

[G-M] W. T. Gowers and B. Maurey, The unconditional basis sequence problem, J. Amer. Math. Soc. 6 (1993), 851-874.

[H-K-L] L. Harrington, A. S. Kechris and A. Louveau, A Glimm-Effros dichotomy for Borel equivalence relations, J. Amer. Math. Soc. 3 (1990), 903-927.

[H] W. Hurewicz, Zur Theorie der analytischen Mengen, Fund. Math. 15 (1930), $4-16$.

[J1] R. C. James, A separable somewhat reflexive Banach space with nonseparable dual, Bull. Amer. Math. Soc. 80 (1974), 738-743.

[J2] - Bases and reflexivity of Banach spaces, Ann. of Math. 52 (1950), 518-527.

[Ka1] R. Kaufman, Topics on analytic sets, Fund. Math. 139 (1991), 215-229.

[Ka2] - Fourier transforms and descriptive set theory, Mathematika 31 (1984), 336-339.

[K] A. S. Kechris, Classical Descriptive Set Theory, Springer, New York, 1995.

[K-L1] A. S. Kechris and A. Louveau, Descriptive Set Theory and the Structure of Sets of Uniqueness, London Math. Soc. Lecture Note Ser. 128, Cambridge Univ. Press, 1987.

[K-L2] -, 一, A classification of Baire class 1 functions, Trans. Amer. Math. Soc. 318 (1990), 209-236.

[Ko] A. Komisarski, Some descriptive set-theoretic properties of the isomorphism relation between Banach spaces, Proc. Amer. Math. Soc. 129 (2001), 3085-3090.

[Kw] S. Kwapien, Isomorphic characterizations of inner product spaces by orthogonal series with vector coefficients, Studia Math. 44 (1972), 583-595.

[La] G. Lancien, Théorie de l'indice et problèmes de renormage en géométrie des espaces de Banach, thèse, Univ. Paris 6, 1992.

[La1] - Dentability indices and locally uniformly convex renormings, Rocky Mountain J. Math. 23 (1993), 635-647.

[La2] -, On the Szlenk index and the weak* dentability index, Quart. J. Math. Oxford (2) 47 (1996), 59-71.

[La3] - Indices de Szlenk et espaces de James, in: Séminaire d'Initiation à l'Analyse, 1989-1990, exp. 28, Univ. Paris VI.

[Lo] H. P. Lotz, The Radon-Nikodym property in Banach lattices, preprint, 1975.

[L-S] J. Lindenstrauss and C. Stegall, Examples of separable spaces which do not contain $\ell_{1}$ and whose duals are non-separable, Studia Math. 54 (1975), 81-105.

[L-T1] J. Lindenstrauss and L. Tzafriri, Classical Banach spaces I. Sequence Spaces, Springer, 1977.

[M] S. Mazurkiewicz, Über die Menge der differenzierbaren Funktionen, Fund. Math. 27 (1936), 244-249.

[Mau] R. D. Mauldin, The set of continuous nowhere differentiable functions, Pacific J. Math. 83 (1979), 199-205. 
[N-P] I. Namioka and R. R. Phelps, Banach spaces which are Asplund spaces, Duke Math. J. 42 (1975), 735-750.

[P] A. Pełczyński, Universal bases, Studia Math. 32 (1969), 247-268.

[Se] A. Sersouri, Some properties of the Pisier-Xu interpolation spaces, Colloq. Math. 65 (1993), 43-50.

[Sz] W. Szlenk, The non-existence of a separable reflexive Banach space universal for all separable reflexive Banach spaces, Studia Math. 30 (1968), 53-61.

[Z] M. Zinsmeister, Les dérivations analytiques, in: Séminaire de Probabilités XXIII, Lecture Notes in Math. 1372, Springer, 1989, 21-46.

Université Paris 6, Équipe d'Analyse

Boîte 186, 4 place Jussieu

75252 Paris Cedex 05, France

Received 15 April 2001 